\title{
Autocatalytic sets in E. coli metabolism
}

\author{
Filipa L Sousa ${ }^{1 *}$, Wim Hordijk² ${ }^{2}$ Mike Steel ${ }^{3}$ and William F Martin ${ }^{1}$
}

\begin{abstract}
Background: A central unsolved problem in early evolution concerns self-organization towards higher complexity in chemical reaction networks. In theory, autocatalytic sets have useful properties to help model such transitions. Autocatalytic sets are chemical reaction systems in which molecules belonging to the set catalyze the synthesis of other members of the set. Given an external supply of starting molecules - the food set - and the conditions that (i) all reactions are catalyzed by at least one molecule, and (ii) each molecule can be constructed from the food set by a sequence of reactions, the system becomes a reflexively autocatalytic food-generated network (RAF set). Autocatalytic networks and RAFs have been studied extensively as mathematical models for understanding the properties and parameters that influence self-organizational tendencies. However, despite their appeal, the relevance of RAFs for real biochemical networks that exist in nature has, so far, remained virtually unexplored.

Results: Here we investigate the best-studied metabolic network, that of Escherichia coli, for the existence of RAFs. We find that the largest RAF encompasses almost the entire E. coli cytosolic reaction network. We systematically study its structure by considering the impact of removing catalysts or reactions. We show that, without biological knowledge, finding the minimum food set that maintains a given RAF is NP-complete. We apply a randomized algorithm to find (approximately) smallest subsets of the food set that suffice to sustain the original RAF.

Conclusions: The existence of RAF sets within a microbial metabolic network indicates that RAFs capture properties germane to biological organization at the level of single cells. Moreover, the interdependency between the different metabolic modules, especially concerning cofactor biosynthesis, points to the important role of spontaneous (non-enzymatic) reactions in the context of early evolution.
\end{abstract}

Keywords: Autocatalytic networks, Origin of life, Metabolic network

\section{Background}

Autocatalytic sets were initially proposed as chemical reaction networks with intrinsic properties that could promote a natural rudimentary selection process en route towards self organization in chemical evolution [1-4]. Until now, autocatalytic sets were mostly theoretical constructs, with only a few artificially designed and constructed examples in real chemistry [5-10]. However, with one exception [11], actual (evolved) biological networks have not been studied explicitly in the context of autocatalytic sets. Here, we take a step in this direction. In particular, we apply a formal framework for autocatalytic sets, known as RAF theory (see Experimental

\footnotetext{
${ }^{*}$ Correspondence: Filipa.Sousa@hhu.de

1 Institute of Molecular Evolution, Heinrich Heine Universität, Düsseldorf, Germany

Full list of author information is available at the end of the article
}

below), to the best-studied metabolic network, that of E. coli.

In order to perform such an analysis, we had to modify the available $E$. coli metabolic network data [12] to conform to the formal RAF framework. For example, since most of the metabolic reactions dealing with carbon and energy metabolism occur within the cytoplasm, the periplasmic reactions as well as transport reactions between the environment, periplasm and cytoplasm were discarded. The exceptions are a few reactions annotated as oxidative phosphorylation, for example cytochrome $c$ reduction and oxidation, which were kept. Also, to apply the RAF algorithm to E. coli metabolism, the network has to be expressed in terms of molecules-reactionscatalysts and, when possible, having the catalyst of each reaction expressed in terms of the cofactors present in the enzyme that catalyzes the reaction. In our definition, 
we include as cofactors all non-protein chemical compounds that are present and/or assist a biochemical transformation. Thus, metal ions, iron-sulfur centers, as well as organic molecules such as flavins or quinones were considered as cofactors. Moreover, if a cofactor like the ones above mentioned is a reactant within a chemical reaction, it would be considered a catalyst of that reaction as well. We did not make any distinction between quinone types, flavin-species or NAD-species partially because of lack of information within annotations and also due to possible promiscuity between the use of analog cofactors. This approach is in agreement with the view that cofactors themselves, metals or even simple amino acids were the initial catalysts of biological reactions [13-19]. This principle can be illustrated with the example of the cofactor pyridoxal phosphate (PLP): in a comparison of 2-aminoisobutyrate decarboxylation reactions catalyzed by a PLP-dependent enzyme, the enzyme-PLP complex was shown to increase the reaction rate $10^{18}$-fold, whereas in the absence of the enzyme, PLP alone increased the rate of decarboxylation by $10^{10}$-fold [20]. As for invoking the role of metals as early catalysts, the continuous abiotic production of methane and formate by serpentinization reactions shows a common trail that connects biology with geochemical occurring reactions whose metal-based "catalysts" embedded in minerals resemble the metal centers found in modern enzymes [21]. This support the view shared by many of the important role of metals in early evolution $[14,16,22,23]$.

Having thus prepared the E. coli metabolic network for analysis, we applied the RAF framework to search for autocatalytic sets within it, studied their structure, and performed sensitivity analyses in terms of importance of individual molecules, reactions, and catalysts.

\section{Experimental}

\section{Autocatalytic sets}

The concept of autocatalytic sets was introduced several decades ago [2-4], and formalized more recently with RAF theory [24-26]. It aims to model life as a functionally closed, self-sustaining reaction system. We briefly review the main definitions and results of RAF theory here.

First, a chemical reaction system (CRS) is defined as a tuple $Q=\{X, \mathcal{R}, C\}$ consisting of a set of chemical species (molecule types) $X$, a set of chemical reactions $\mathcal{R}$, and a catalysis set $C$ indicating which molecule types catalyze which reactions. Next, the notion of a food set $F \subset X$ is included, which is a subset of molecule types that are assumed to be freely available from the environment. Finally, an autocatalytic set (or RAF set) is defined as a subset $\mathcal{R}^{\prime} \subseteq \mathcal{R}$ of reactions (and associated molecule types) which is:
1. Reflexively Autocatalytic (RA): each reaction $r \in \mathcal{R}^{\prime}$ is catalyzed by at least one molecule type involved in $\mathcal{R}^{\prime}$, and

2. Food-generated (F): all reactants in $\mathcal{R}^{\prime}$ can be created from the food set $F$ by using a series of reactions only from $\mathcal{R}^{\prime}$ itself.

This definition captures the notion of a functionally closed (RA) and self-sustaining (F) reaction network. A formal mathematical definition of RAF sets is provided in $[25,27]$, including an algorithm for finding RAF sets in a general CRS. This algorithm has a worst-case running time of $O\left(|R|^{2} \log |R|\right)$, i.e., it is efficient (polynomial in the size of the full reaction network). In previous work, we have applied the RAF algorithm to random reaction networks with up to several millions of reactions, on which the average running time was sub-quadratic [25].

A CRS may not contain any RAF, but when it does it always contains a unique maximal RAF (maxRAF), and this maxRAF is the one the RAF algorithm finds. Moreover, it has been shown that a maxRAF can often be decomposed into several smaller subsets which themselves are RAF sets (subRAFs) [28]. If such a subRAF cannot be reduced any further without losing the RAF property, it is referred to as an irreducible RAF (irrRAF). The existence of multiple autocatalytic subsets can actually give rise to an evolutionary process [5], and the emergence of larger and larger autocatalytic sets over time [28]. In this paper we will also consider a more restrictive type of autocatalytic set called a "constructible" autocatalytic set (CAF set) [29]. This is an RAF set can be dynamically realized without any of its reactions having to happen "spontaneously" (uncatalyzed) initially to get all catalysts present in the system.

A simple example of a CRS and its RAF (sub)sets, using the well-known binary polymer model, is given in Figure 1 . In the binary polymer model $[3,4]$, molecules are represented by bit strings up to a given length $n$, and the possible reactions are ligation and cleavage. In a ligation reaction, two bit strings are "glued" together into a longer bit string, e.g., $00+111 \rightarrow 00111$. In a cleavage reaction, a bit string is "cut" into two smaller substrings, e.g., $101010 \rightarrow 1010+10$. Finally, the bit strings are assigned randomly as catalysts to the possible reactions according to a given probability of catalysis $p$ (which is the probability that an arbitrary bit string catalyzes an arbitrary reaction).

Figure 1(a) shows an instance of the binary polymer model with $n=5, p=0.0045$, and a food set consisting of all bit strings of length one and two. Only the catalyzed reactions (12 in total) are shown. Black dots indicate molecule types (not labeled), and white boxes indicate reactions. Solid black arrows indicate molecules going into and coming out of reactions, and dashed grey arrows 


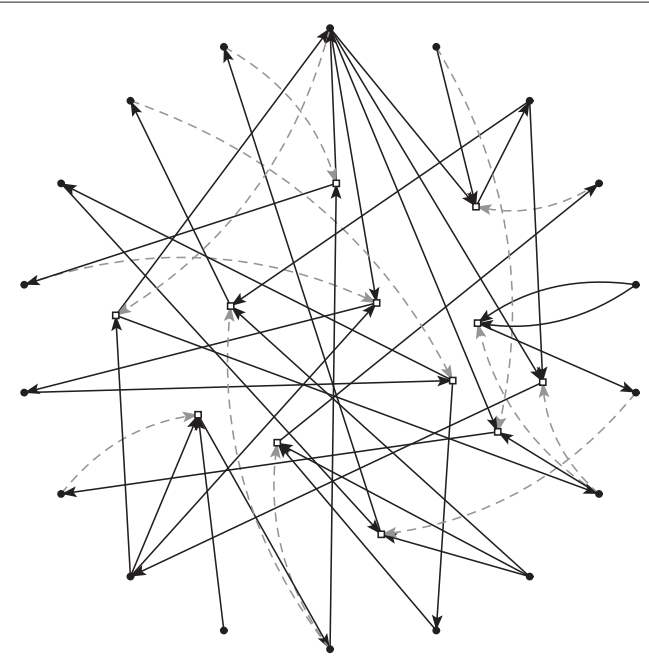

(a)

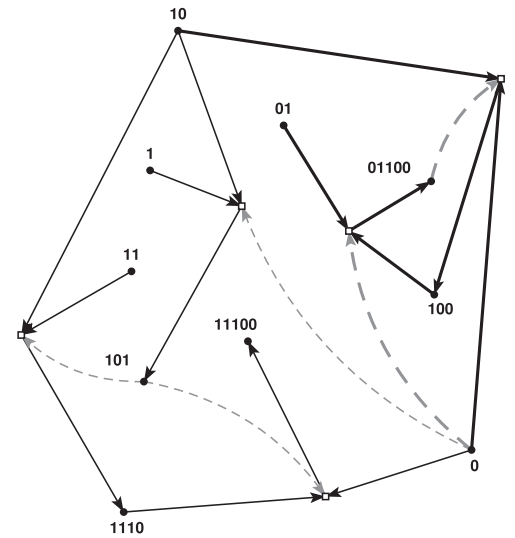

(b)

Figure 1 An example CRS and its (sub)RAFs. (a) An instance of the binary polymer model (catalyzed reactions only). Black dots (on the outside, around a circle) indicate molecule types (not labeled), and white boxes (inside the circle) indicate reactions. Solid black arrows indicate molecules going into and coming out of reactions, and dashed grey arrows indicate catalysis. (b) The maxRAF as found by applying the RAF algorithm to the CRS in (a). This maxRAF contains an irreducible RAF of two reactions (indicated by the bold arrows). The food set consists of the bit strings of length one and two.

indicate catalysis. Applying the RAF algorithm to this CRS results in the maxRAF shown in Figure 1(b), which consists of five reactions. Furthermore, the maxRAF contains an irreducible RAF of two reactions (indicated by the bold arrows). Note that neither the maxRAF nor this irrRAF is a CAF, as one of the two reactions in the irrRAF needs to happen spontaneously initially before the full RAF set can be realized dynamically.

Using the binary polymer model, it was shown that RAF sets are highly likely to exist, even for very moderate levels of catalysis (between one and two reactions catalyzed per molecule, on average) $[25,26,29,30]$. Moreover, this result still holds under various model extensions, such as a more realistic "template-based" form of catalysis $[27,31,32]$, a power law distribution of catalysis [33], and even non-polymer systems [34].

In [28] it was shown that, in principle, there can be an exponentially large number of irrRAFs within a given maxRAF. So, there is no hope of efficiently enumerating all irrRAFs within an arbitrary RAF set. Furthermore, in [35] it was shown that even finding the smallest irrRAF is NPcomplete. However, the RAF algorithm can be extended to provide a method to randomly sample irrRAFs from a given RAF set $\mathcal{R}^{\prime}$, as follows [35]:

\section{irrRAF sampling algorithm}

1. Randomly reorder the list of reactions contained in $\mathcal{R}^{\prime}$.

2. For each next reaction $r_{i} \in \mathcal{R}^{\prime}$ do:

(a) Remove $r_{i}$ from $\mathcal{R}^{\prime}$. (b) Apply the RAF algorithm to $\mathcal{R}^{\prime}$.

(c) If the resulting (maximal) RAF set $\mathcal{R}^{\prime \prime}$ is non-empty, set $\mathcal{R}^{\prime}=\mathcal{R}^{\prime \prime}$, otherwise return $r_{i}$ to $\mathcal{R}^{\prime}$.

3. Return the irreducible RAF set $\mathcal{R}^{\prime}$.

Sampling irrRAFs, and also keeping track of the sizes of the intermediate subRAFs while iterating step 2, can provide useful insight into the modularity of RAF sets, as we show in our results below.

Finally, real autocatalytic sets have actually been constructed in laboratory experiments [6-10]. Recently it was shown that the formal RAF framework can be directly applied to such real chemical systems, not only reproducing the experimental results, but also providing predictions about the system's behavior that would be difficult to obtain from the chemical experiments alone [36]. However, these examples, although real, have all been carefully designed and created in controlled experiments. Here, we take a first step at applying the formal RAF framework to a biological CRS: the metabolic network of $E$. coli.

\section{The metabolic network of $E$. coli}

All reactions, their functional annotation, and information about the gene product(s) that catalyze them, were retrieved from the most recent $E$. coli metabolic network data set [12]. Each E. coli gene was parsed with the UNIPROT information available on 6 March 2013 regarding the type of metals and cofactors [37]. 
For the purpose of identifying RAF sets within the $E$. coli metabolic network, the following transformations of the metabolic network were performed: i) Transport reactions and reactions localized within the E. coli periplasm (except those involved in oxidative phosphorylation) are removed from the network, and the affected molecules are included in the food set. Thus, molecules taken in from the environment (indicated as " $\operatorname{xxx}[\mathrm{e}]$ " in the original data set) are directly available as food molecules; ii) In reactions catalyzed by a protein that uses a cofactor or metals, these cofactors and metals are assigned as the catalysts for that reaction. This has the effect of integrating organic cofactors into the reaction network. All metals are included in the food set (unless they are organized as FeS clusters, in which case they are treated as synthesized organic cofactors); iii) Reactions catalyzed by a protein that has no known or annotated cofactor are defined as being catalyzed by a general catalyst called "Protein", which is included in the food set. In case of RNAdependent reactions, a generic "RNA" catalyst was introduced that also belongs to the food set. This has the effect of keeping cofactor-independent reactions within the set of catalyzed reactions. Moreover, by grouping these reactions with the same generic catalyst (Protein or RNA), we are simplifying the network's catalyst space without losing biological information. iv) Reactions for which the $E$. coli enzyme is unknown were assigned to another general catalyst called "genCat", which is also included in the food set, to resolve incomplete data; v) Groups of catalysts with common properties and common biosynthetic pathways, such as menaquinone/ubiquinone, NAD/NADP, or flavins, are grouped together into a "pool" of equivalent catalysts (see Table 1); vi) Bi-directional reactions are split up into two separate reactions, one forward and one reverse, but catalyzed by the same catalyst. This does not affect the network structure in any way, but in some cases makes it more amenable to the RAF analysis; vii) If a reaction requires more than one catalytic molecule and all catalysts need to be present simultaneously, an additional reaction is included that creates a "catalyst compound" from these individual catalysts, which then catalyzes the given reaction. The reaction that creates such a compound is catalyzed by a general catalyst called " $\mathrm{X}$ ". These reactions are annotated as Catalyst reactions and do not exist in $E$. coli's biological metabolic network. This has the effect of allowing several cofactors to be required for a reaction to take place. This transformation is required for the RAF algorithm to work without changing the biological aspect of the network; viii) All reactions where cofactors (e.g., quinones, metals, FAD) were required by a component of the enzyme, whether involved in catalysis or not, have these cofactors in their required catalyst list. This has the effect of making the reactions dependent on molecules like quinones; ix) If a reaction can be
Table 1 The different catalyst pools

\begin{tabular}{|c|c|}
\hline Cofactor & Abbreviation/Group \\
\hline Thiamine pyrophosphate/Thiamin & Thiamin \\
\hline $\mathrm{NAD}^{+} / \mathrm{NADH} ; \mathrm{NADP}^{+} / \mathrm{NADPH}$ & nad-pool \\
\hline Pyridoxal phosphate & pydx5p \\
\hline Pyridoxal & pydx \\
\hline Lipoamide & lipoamp \\
\hline Methylcobalamin/Cobalamin & Cob \\
\hline Coenzyme A and derivates & coa \\
\hline Tetrahydrofolic acid and derivates & folate \\
\hline Menaquinone/Ubiquinone & Q \\
\hline Pyrroloquinoline quinone & PQQ \\
\hline Topaquinone & topaquinone \\
\hline FMN/FMNH 2 and FAD/FADH ${ }_{2}$ and Riboflavin & Flavins \\
\hline Glutathione oxidized and reduced & Glutathione \\
\hline S-Adenosyl methionine & SAM \\
\hline Siroheme & sheme \\
\hline Heme B/Heme O & Heme \\
\hline Heme D & HemeD \\
\hline All tRNA & RNA \\
\hline Molybdopterin & Molybdopterin \\
\hline $4 \mathrm{Fe}-4 \mathrm{~S} ; 2 \mathrm{Fe}-2 \mathrm{~S} ; 3 \mathrm{Fe}-4 \mathrm{~S}$ & Iron-Sulfur-cluster \\
\hline Divalent-cations & Divalent-cations \\
\hline
\end{tabular}

independently catalyzed by two or more proteins, all possible pairs "reactions:catalyst" are included, each instance being catalyzed by the cofactors present in the respective protein; $\mathrm{x}$ ) When the type of metal was not specified, we assumed that any of the divalent-metal ions could catalyze the reaction, and a pool of divalent metal ions is included in the food set. This allows plasticity and mimics biological enzymes.

Finally, xi) reactions annotated in [12] as uncatalyzed were assigned a general catalyst called "spont" (included in the food set). We thus assume that these reactions still happen at a high enough rate to be considered relevant. This approximation might seem counter intuitive since in the strict formalism of RAFs, uncatalyzed reactions are outlawed. However, within a cell, several uncatalyzed reactions do occur, at rates high enough not to impair its metabolic function. Prevailing uncatalyzed reactions within autocatalytic sets tend to give rise to molecules that are not members of the set itself but can be incorporated into it, allowing the evolution of new autocatalytic sets $[4,38]$. Thus, uncatalyzed biological reactions are processes that introduce chemical species (or increase their availability) in the cell's environment. In practical terms, and since the generic catalysts are part of the food set, including spontaneous 
reactions in the network by the insertion of the generic catalyst "spont" is similar to the introduction of the products of these reactions in the food set itself, as long as their substrates are available. In reality, and excluding the cases were energy coupling exists (e.g. electron bifurcation [39] or Q-cycle [40]) catalyzed reactions are no more than spontaneous uncatalyzed reactions whose activation energy is lowered by the action of a catalyst. In the scenario of early evolution, the primordial reactions would have occurred spontaneously with the help of metal ions and simple abiotic cofactors, with protein dependent catalytic reactions appearing later as add-ons [41]. However, the removal of this generic catalyst does not have high impact on the size of the E. coli metabolic RAF (see below).

The initial food set thus consists of all molecules exchanged with the environment. This includes the 324 molecule types labeled as " $\operatorname{xxx}[\mathrm{e}]$ " in the original data set, plus the ones produced in the periplasm, the introduced general catalysts, and a few essential species such as ATP, to make a total of 438 food molecules. The resulting reaction network, consists of 1199 distinct molecules types (including the 438 food molecules), 1826 reactions (belonging to 33 different functional categories) and 42 catalysts. We then applied the RAF framework to analyze this CRS for the existence and structure of autocatalytic sets.

A second food set was created based on the laboratory conditions given for E. coli growth on glucose-6phosphate and glucose as in [42]. The reactions included in the resulting RAF network were mapped to E. coli Kegg pathways [43]. Hierarchical clustering and plotting of this network were performed in MATLAB.

\section{Results and discussion}

RAF sets in the metabolic network of $E$. coli

Applying the RAF algorithm to the $E$. coli metabolic network results in a maximal RAF set (maxRAF) consisting of 1787 reactions. This corresponds to $98 \%$ of the full 1826-reaction metabolic network, that is, only 39 reactions of the full network are not part of the maxRAF. When ATP or an equivalent compound such as ADP is available in this food set, the resulting RAF set is also a socalled "constructible" autocatalytic set (CAF set). This is in agreement with previous results from [11] where ATP was identified as an obligate autocatalytic metabolite in all metabolic networks studied.

An outline of the number of reactions per functional category in the maxRAF is presented in Table 2, where it can be seen that all the major functional categories, such as amino acid biosynthesis, carbon metabolism, and cofactor and prosthetic group biosynthesis, are represented within the RAF set.
Table 2 The number of reactions in the maxRAF set belonging to each functional category

\begin{tabular}{|c|c|}
\hline Functional category & Reactions \\
\hline Alanine and Aspartate Metabolism & 11 \\
\hline Alternate Carbon Metabolism & 217 \\
\hline Anaplerotic Reactions & 11 \\
\hline Arginine and Proline Metabolism & 45 \\
\hline Cell Envelope Biosynthesis & 135 \\
\hline Citric Acid Cycle & 23 \\
\hline Cofactor and Prosthetic Group Biosynthesis & 235 \\
\hline Cysteine Metabolism & 13 \\
\hline Folate Metabolism & 11 \\
\hline Glutamate Metabolism & 6 \\
\hline Glycerophospholipid Metabolism & 150 \\
\hline Glycine and Serine Metabolism & 17 \\
\hline Glycolysis/Gluconeogenesis & 34 \\
\hline Glyoxylate Metabolism & 4 \\
\hline Histidine Metabolism & 12 \\
\hline Inorganic Ion Metabolism & 32 \\
\hline Lipopolysaccharide Biosynthesis / Recycling & 39 \\
\hline Membrane Lipid Metabolism & 78 \\
\hline Methionine Metabolism & 16 \\
\hline Methylglyoxal Metabolism & 10 \\
\hline Murein Recycling & 20 \\
\hline Nitrogen Metabolism & 13 \\
\hline Nucleotide Salvage Pathway & 173 \\
\hline Oxidative Phosphorylation & 65 \\
\hline Pentose Phosphate Pathway & 19 \\
\hline Purine and Pyrimidine Biosynthesis & 35 \\
\hline Pyruvate Metabolism & 23 \\
\hline Threonine and Lysine Metabolism & 25 \\
\hline Tyrosine, Tryptophan, and Phenylalanine Metabolism & 29 \\
\hline Unassigned & 21 \\
\hline Valine, Leucine, and Isoleucine Metabolism & 23 \\
\hline tRNA Charging & 23 \\
\hline Catalysts Reaction & 219 \\
\hline Total & 1787 \\
\hline
\end{tabular}

\section{Minimum food set}

A crucial determinant for the existence (and size) of RAF sets is the composition of the food set. As described above, the initial food set for the $E$. coli metabolic network consists of 438 molecule types, 324 of these being chemical species that are exchanged with the environment in [12]. A large redundancy is found within this last group, namely in terms of redox state of the uptaken metals or interconvertible chemical pairs 
(e.g. N-acetyl-D-galactosamine and N-acetyl-D-galactosamine 1-phosphate). Nevertheless, they provide a good starting point for this analysis. The additional molecules of the food set are those produced in the periplasm, the introduced general catalysts, and a few essential molecules such as ATP. Although ATP is produced by the reaction system, it needs to be part of the food set, because otherwise the reaction system does not move forward initially. This role of ATP in biological networks has already been pointed out in [11] where it was shown that regardless of the initial food set, ATP or equivalent compounds participating in the same autocatalytic cycle are obligatory autocatalytic metabolites. In the context of early evolution, this can be seen as a requirement for favorable thermodynamics in spontaneous chemical evolution [44-46].

However, this initial food set can be reduced without diminishing the size of the maximal RAF set. Out of the 438 initial food molecules, there are 117 "essential" ones: removing any one of these individually reduces the size of the maximal RAF set. In the majority of cases (103) the RAF set is reduced by less than 30 reactions, but there are 7 molecules that reduce the size of the RAF set by more than 1000 reactions when removed from the food set, in particular the generic catalysts.

Removing the remaining 321 molecules from the food set, i.e., using only the 117 essential food molecules, also results in a reduced maxRAF. So, at least some subset of these 321 molecules needs to remain in the food set to maintain the maxRAF. This brings up the question of whether it is possible to (efficiently) find a minimum food set that maintains a given RAF set in a reaction network. Unfortunately, this problem turns out to be NP-complete, as the following theorem states.

Consider the following combinatorial optimization problems.

\section{min-F RAF:}

INSTANCE: A CRS $\mathcal{Q}=(X, \mathcal{R}, C)$, and food set $F \subseteq X$, with $\mathcal{R}^{\prime} \subseteq \mathcal{R}$ an $\operatorname{RAF}$ for $(\mathcal{Q}, F)$, and a positive integer $k$.

QUESTION: Is there a subset $F^{\prime}$ of $F$ of size at most $k$ for which $\mathcal{R}^{\prime}$ is an $\operatorname{RAF}$ for $\left(\mathcal{Q}, F^{\prime}\right)$ ?

\section{min-F generation:}

INSTANCE: A set of reactions $\mathcal{R}^{\prime}$ that is $F$-generated, and a positive integer $k$.

QUESTION: Is there a subset $F^{\prime}$ of $F$ of size at most $k$ for which $\mathcal{R}^{\prime}$ is $F^{\prime}$-generated?

Recall that $F$-generated means that each reactant in $\mathcal{R}^{\prime}$ is either present in $F$ or can be created from $F$ by using a series of reactions only from $\mathcal{R}^{\prime}$ itself.
Theorem 1. The min-F RAF and min-F generation problems are NP-complete.

Proof. See the Appendix.

This seems a rather technical result, but it implies that there is no hope of constructing an efficient (polynomialtime) algorithm for finding a minimum food set to maintain the same maxRAF set. This, therefore, presents a limit to our ability to study a given reaction network analytically.

However, we can still take a heuristic approach and construct a randomized search algorithm to sample food subsets, and then take the smallest set from the resulting samples as an approximate solution to the min-F problem. This randomized algorithm is similar to that for sampling irreducible RAF sets as described in section "Autocatalytic sets", and analogous to the method used in [47] to find minimal metabolic networks.

\section{min-F search algorithm}

1. Randomly reorder the list of molecule types the original food set $F$.

2. For each next element $f_{i} \in F$ do:

(a) Remove $f_{i}$ from $F$.

(b) Apply the RAF algorithm.

(c) If the resulting (maximal) RAF set is smaller than before, return $f_{i}$ to $F$, otherwise leave it out.

\section{Return the (reduced) food set $F$.}

Repeating this algorithm any number of times on the $E$. coli data set always returns the same smallest size for the food set of 123 molecules. This includes the 117 essential molecules, plus some combination of six molecule types from the remaining 321 compounds. This combination of six molecule types is not always the same, though, but they do come from a very small subset. Table 3 shows an example of ten possible combinations found by our randomized search algorithm.

Table 3 reveals the presence of groups of molecules such as adocbl (adenosyl-cobalamin) and cbl1 (cobalamin), or atp/adp/gtp. These grouped molecules correspond to equivalent metabolites in the sense that they can break down the same autocatalytic cycle [11] and the presence of any molecule from each of these groups in the food set is sufficient to maintain the original maxRAF. Thus, even though the general problem of finding the minimum food set is NP-complete, in case of the E. coli network it seems very likely that the minimum food set is of size 123 , but that it is not unique. 
Table 3 Ten different combinations of the additional six food molecules necessary to maintain the maximal RAF set

\begin{tabular}{|c|c|c|c|c|c|c|c|c|c|c|}
\hline Molecule & 1 & 2 & 3 & 4 & 5 & 6 & 7 & 8 & 9 & 10 \\
\hline ATP & $x$ & & & & & & $x$ & & & \\
\hline Cob(I)alamin & $x$ & & & & & & & & $x$ & \\
\hline fructoselysine & $x$ & $x$ & & $x$ & & $x$ & & $x$ & $x$ & \\
\hline D-Fructuronate & $x$ & & & $x$ & $x$ & & & $x$ & & \\
\hline D-Gluconate & $x$ & & & & & $x$ & & $x$ & & \\
\hline $\mathrm{SO}_{2}$ & $x$ & $x$ & & & $x$ & $x$ & $x$ & $x$ & & \\
\hline Adenosylcobalamin & & $x$ & $x$ & $x$ & $x$ & $x$ & $x$ & $x$ & & $x$ \\
\hline ADP & & $x$ & & $x$ & & & & $x$ & $x$ & \\
\hline D-Glucuronate & & $x$ & $x$ & & & $x$ & $x$ & & $x$ & $x$ \\
\hline L-Idonate & & $x$ & $x$ & $x$ & $x$ & & $x$ & & & $x$ \\
\hline GTP & & & $x$ & & $x$ & $x$ & & & & $x$ \\
\hline $\mathrm{H}_{2} \mathrm{O}_{2}$ & & & $x$ & & & & & & $x$ & $x$ \\
\hline psicoselysine & & & $x$ & & $x$ & & $x$ & & & $x$ \\
\hline $\mathrm{O}_{2}$ & & & & $x$ & & & & & & \\
\hline 5-Dehydro-D-gluconate & & & & & & & & & $x$ & \\
\hline
\end{tabular}

With such a minimum food set, however, the maxRAF set is not "constructible" anymore (it would need to have some catalyst-requiring reactions occurring without catalyst to begin with untill all catalysts have been generated), although there is still a CAF subset of 434 reactions within the maximal RAF. For the remainder of our analysis, we use the essential 117 molecules plus the first set of six additional molecules from Table 3 as the (minimum) food set.

This large number of "essential" molecules in the food set is to some extent an artifact arisen from how the initial metabolic network was constructed [12] and from the condition we imposed that the size of the maximal RAF should not be reduced. Most of the essential food molecules correspond to specifications of generic compounds involved in the peripheral glycerophospholipid metabolism (seven derivatives each of 2-acyl-sn-glycero-3-phosphoethanolamine, 2acyl-sn-glycero-3-phosphoglycerol and enoyl-sn-glycerol 3-phosphate), post-translationally modified amino acids or configurations of usually rare biological isomers that participate in few reactions (five post-translationally modified amino acids, one D-aminoacid, five L-sugars and D- and L-tartaric acid). In all of these cases, no reaction(s) exist for their synthesis in the network although they participate as substrates. For the same reason, additional molecules such as lipoate, dopamine, ferric 2,3dihydroxybenzoylserine or Fe(III) hydroxamic acid, also need to be included in the food set as essential molecules.

Another factor contributing to the large number of food molecules are environmental adaptations of E. coli. As recently summarized by Mackie et al. [42] E. coli can grow under different conditions, for example, under aerobic and anaerobic conditions, or using different carbon sources.
The extended food set allows for all of these alternative pathways to be functioning within the RAF network. This raises the question of why to use E. coli as a model organism for probing questions regarding self-organization of primordial metabolism. The reason is simple: even though other metabolic networks exist [48,49], E. coli's is the best studied and annotated biological network available, even if not perfect (see Conclusions). Finally, five generic catalysts contribute to the large number of food set molecules. These are artificial constructs within the network and both their impact and biological significance within the RAF differ (see below). Additional molecules such as thiamin also needed to be included in the food set as essential molecules, although within the metabolic network, reactions for thiamin synthesis do exist. This apparently counterintuitive measure has two reasons. First, our network does not contain any biosynthetic route for the synthesis of 4-methyl-5-(2-hydroxyethyl)-thiazole, a thiamin precursor involved in the pyrimidine branch of thiamin biosynthesis. Second, even if this molecule is included in the food set, the double autocatalytic nature of the thiazole branch of thiamin biosynthesis in E. coli would prevent these reactions to be included in the RAF set. Briefly, the enzymes involved in the biosynthesis of thiamin are dependent, among other cofactors, on PLP [50] and thiamin itself [51]. On the other hand, the biosynthesis of PLP, besides being PLP dependent [52], has one step that is thiamin dependent [51]. Thus, in order to include these reactions within the RAF system, several reactions would have to proceed uncatalyzed or one of the cofactors would have to be present in the food-set. This atributte suggests that at the origin of life, some forms of the cofactors existed, hence were synthesized 
spontaneously, before their biosynthetic pathway arose. The remaining essential molecules of this food set, consist mainly of 20 different metals, nine inorganic compounds including gases, 28 distinct carbon and sulphur sources, and ATP. The full list of this food set is presented in the Additional file 1.

\section{The role of catalysts}

Out of the 1199 molecule types in the network, only 42 (3.5\%) act as catalysts, either by themselves or as part of a catalyst compound. Thus, in the E. coli metabolic network, most molecules do not catalyze any reactions at all, some catalyze a few reactions, and there are a few molecules that catalyze many reactions.

Table 4 lists all 42 catalysts, ordered by the number of reactions they catalyze ("cat"; these include reactions they catalyze as part of a composite catalyst). The table also shows by how many reactions the maxRAF is reduced if each of these 42 catalysts is removed individually from the network (labeled as "rem"). As expected, the generic catalysts "Protein" and " $\mathrm{X}$ " affect the RAF set by reducing its size drastically. This is because of the large number of reactions they catalyze (as with "Protein"), or because all reactions catalyzed by them produce other catalysts or groups of catalysts essential for other reactions in the
RAF set (as with "X"). In the current E. coli RAF network, at least $65 \%$ of the reactions are catalyzed by a cofactor and this number would be larger were complete annotations for the genes available (see Conclusions). So, even with a possible bias toward Protein catalyzed reactions, that participate and/or connect different metabolic pathways, we still recall the importance of cofactors within this metabolism. In contrast, the removal of the generic catalyst introduced to allow for uncatalyzed reactions (spont) does not have a significant impact on the size of the E. coli metabolic RAF.

When some catalysts that participate in only a few reactions are removed from the network, a large decrease in the size of the RAF can be observed. For example, 5-phosphoribosyl diphosphate (PRPP) is only a catalyst in the conversion of uracil to UMP (and is a substrate in only 13 reactions), but the removal of this conversion reaction reduces the RAF size by 1377 reactions, even though UMP can be produced by seven other distinct reactions. This shows the central role of PRPP in biological networks. In E. coli, PRPP is involved in many metabolic pathways being the precursor of histidine and NADH. Within this food set, the only possible route for the de novo synthesis of $\mathrm{NADH}$ depends on a reaction where PRPP reacts with quinolinate to form nicotinate

Table 4 The 42 catalysts ordered by the number of reactions they catalyze (cat), also indicating by how many reactions the RAF set is reduced when each catalyst is removed from the network (rem)

\begin{tabular}{|c|c|c|c|c|c|}
\hline & cat & rem & & cat & rem \\
\hline Protein & 582 & 1644 & pyruvate & 9 & 630 \\
\hline nad-pool & 298 & 1353 & Calcium & 9 & 31 \\
\hline X & 185 & 1642 & Cob & 6 & 15 \\
\hline Magnesium & 142 & 1476 & Copper & 5 & 31 \\
\hline flavin & 139 & 1353 & Nickel & 5 & 11 \\
\hline coa & 103 & 619 & sheme & 5 & 7 \\
\hline pydx5p & 90 & 1353 & lipoamp & 4 & 8 \\
\hline Iron-Sulfur-cluster & 86 & 1353 & Potassium & 3 & 166 \\
\hline Zinc & 81 & 1432 & topaquinone & 3 & 5 \\
\hline Divalent-cations & 78 & 1413 & Molybdenium & 10 & 18 \\
\hline Q & 60 & 98 & pan4p & 2 & 625 \\
\hline Iron & 55 & 1353 & dpcoa & 2 & 621 \\
\hline Manganese & 29 & 145 & Glutathione & 2 & 30 \\
\hline Molybdopterin & 28 & 67 & Tungsten & 2 & 11 \\
\hline SAM & 27 & 159 & hemeD & 2 & 5 \\
\hline folate & 24 & 682 & PQQ & 2 & 5 \\
\hline genCat & 23 & 1367 & pydx & 2 & 4 \\
\hline RNA & 21 & 96 & prpp & 1 & 1377 \\
\hline heme & 18 & 31 & $\mathrm{HCO}_{3}$ & 1 & 174 \\
\hline Thiamin & 17 & 1378 & Sodium & 1 & 4 \\
\hline spont & 17 & 42 & Chloride & 1 & 2 \\
\hline
\end{tabular}


D-ribonucleotide. Thus, without PRPP there is no NADH synthesis and the network falls apart.

The extensive biological action of the different cofactors can be observed by the uneven distribution of the catalysts according to their functional annotations, see Figure 2.

\section{Modularity in the RAF set}

The maxRAF contains $98 \%$ of the reactions in the $E$. coli metabolic network. An obvious question arises: "Is there any modularity in this RAF set?"

First, we looked at hierarchical levels of reactions. Starting with the full maxRAF and only the food molecules, we considered all reactions that can proceed catalyzed, i.e., all reactions in the maxRAF that have all their reactants and at least one of their catalysts in the molecule set. This represents the "level 0" reactions. Next we added all the products of these level 0 reactions to the molecule set, and considered all additional reactions that then proceed catalyzed. This represents level 1 (all reactions one reaction step away from the food set). We repeated this procedure for subsequent levels, until the number of reactions that can proceed catalyzed does not further increase. In short, a reaction in level $i$ is $i$ reaction steps away from the food set. Since the maxRAF (with the minimum food set) is not a CAF, obviously there will be some reactions that are not in any of these levels. These are the "non-CAF" reactions.

Applying this analysis to the E. coli maxRAF results in a number of reactions in each level as shown in Table 5 . The

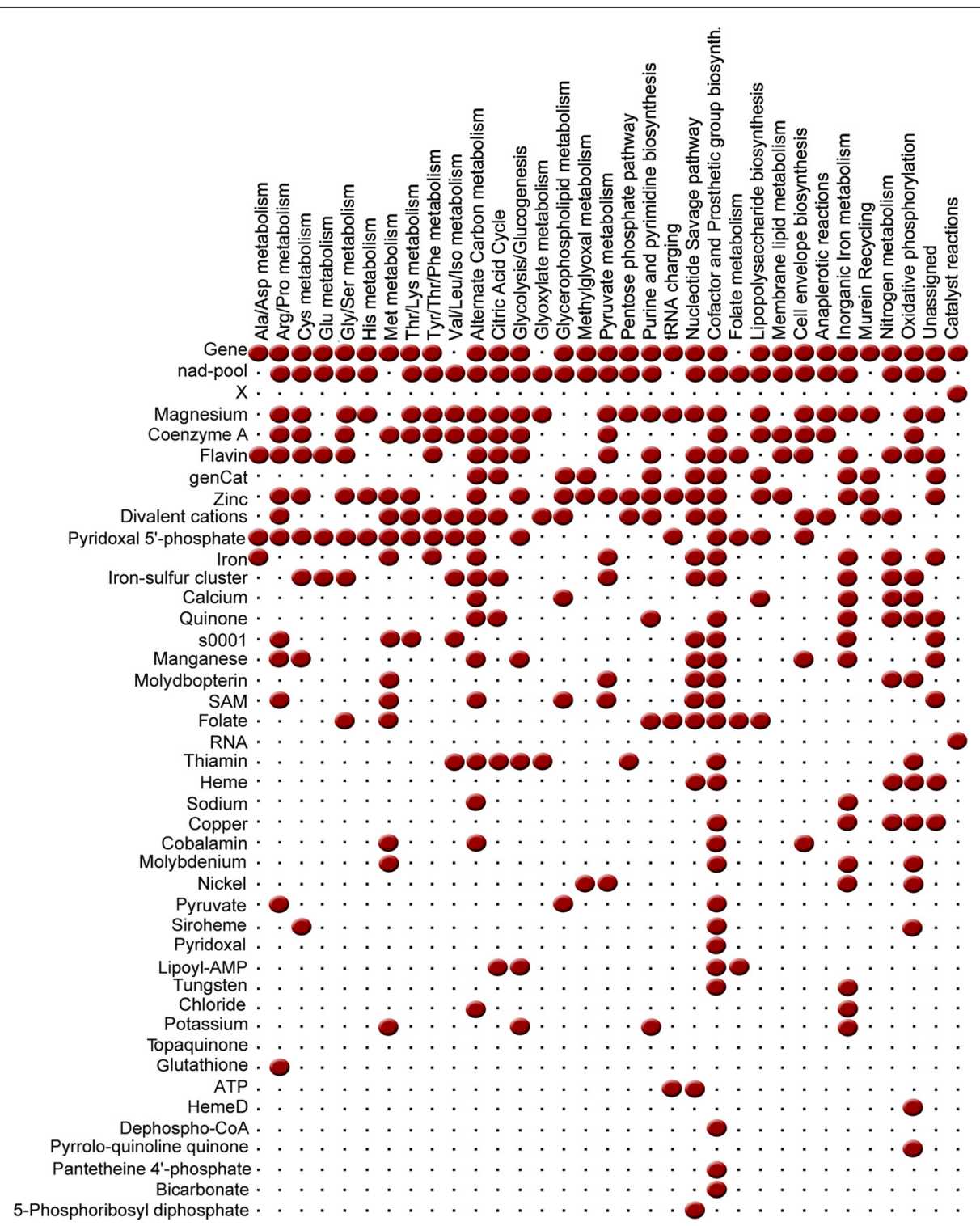

Figure 2 Distribution of the catalysts over the different functional categories. The participation of each one of the catalysts (rows) over the different functional categories (columns) is represented by a red dot. 
Table 5 The number of reactions in each hierarchical level in the maxRAF of $E$. coli

\begin{tabular}{lrrrrrrrr}
\hline level: & 0 & 1 & 2 & 3 & 4 & 5 & 6 & 7 \\
reacs: & 63 & 82 & 102 & 77 & 49 & 16 & 16 & 11 \\
& & & & & & & & \\
level: & 8 & 9 & 10 & 11 & 12 & 13 & 14 & non-CAF \\
reacs: & 7 & 5 & 2 & 1 & 1 & 1 & 1 & 1353 \\
\hline
\end{tabular}

reactions in levels 0 to 14 , taken together, constitute the 434-reaction CAF subset that exists within the maxRAF (as mentioned above). However, most reactions are in the non-CAF level, i.e., at least some of them will need to happen spontaneously (uncatalyzed) at least once before the full maxRAF can come into existence in a dynamical sense (i.e. before all catalyst are present in the system).

Finding a minimum subset of reactions that, when allowed to proceed uncatalyzed at least once, realizes the full maxRAF turns out to be an NP-hard problem [32] (in fact, even just finding the size of such a smallest subset is NP-hard). Therefore, determining the distance (number of reaction steps) from the food set of these non-CAF reactions appears to be intractable.

Another way of looking for modularity in an RAF set is as follows. First, construct a "connectivity graph" $G$ were each node in $G$ corresponds to a reaction in the maxRAF. Next, link reaction $r_{i}$ to reaction $r_{j}$ if a product of $r_{i}$ is either a reactant or a catalyst of $r_{j}$ transforming $G$ in a directed graph. The strongly connected components of this digraph can now be computed.

Constructing $G$ and computing its strongly connected components for the RAF set in the $E$. coli metabolic network results in 93 such components. However, 87 of these are of size one, five are of size two, and one is of size 1690. This indicates that the maxRAF mostly consists of one large connected component showing, as previously pointed out by $[11,13]$, the auto- and cross-catalytic behavior of metabolic networks.

\section{The effect of removing reactions}

Recall that an irreducible RAF set (irrRAF) is an RAF set from which no reactions can be removed without losing the RAF property. Applying the irrRAF sampling algorithm (as described in section "Autocatalytic sets") to the maxRAF in the E. coli metabolic network (using the minimum food set) always results in an irrRAF of size one. This is not surprising, as our hierarchical levels analysis above resulted in 63 reactions in level 0 . Since all of these reactions have their reactants and at least one catalyst in the food set, they are by definition RAF sets by themselves (and, consequently, also irrRAFs, as they are of size one). We refer to these as "trivial" (irr)RAFs. Removing these trivial irrRAFs from the network, however, breaks the original maxRAF, as the resulting RAF set now contains only 17 reactions (with irrRAFs of sizes two and three). So, the "trivial" RAFs are also "essential" for the full maxRAF.

It is still informative, however, to apply the irrRAF sampling algorithm and see how the size of the RAF set is reduced with each (potential) removal of a reaction. Figure 3 shows the sizes of the intermediate RAF sets while iterating the sampling algorithm, for ten repetitions of the method. In most cases, the removal of a reaction reduces the RAF size by only a very small amount and only in few cases this removal results in a significant reduction in the RAF size. For each reaction that reduces the RAF

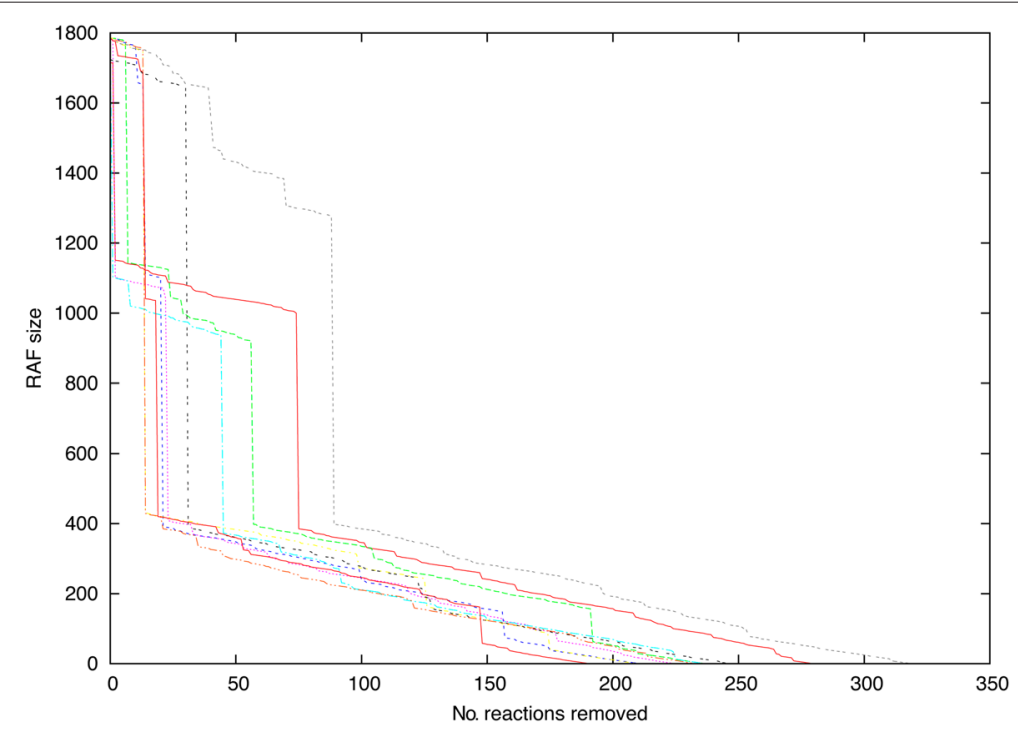

Figure 3 Ten sequences of repeatedly and randomly removing reactions. Thin lines represent the impact of randomly removing a reaction in the RAF size. Each line decrease correspond to the removal of a single reaction from the network. 


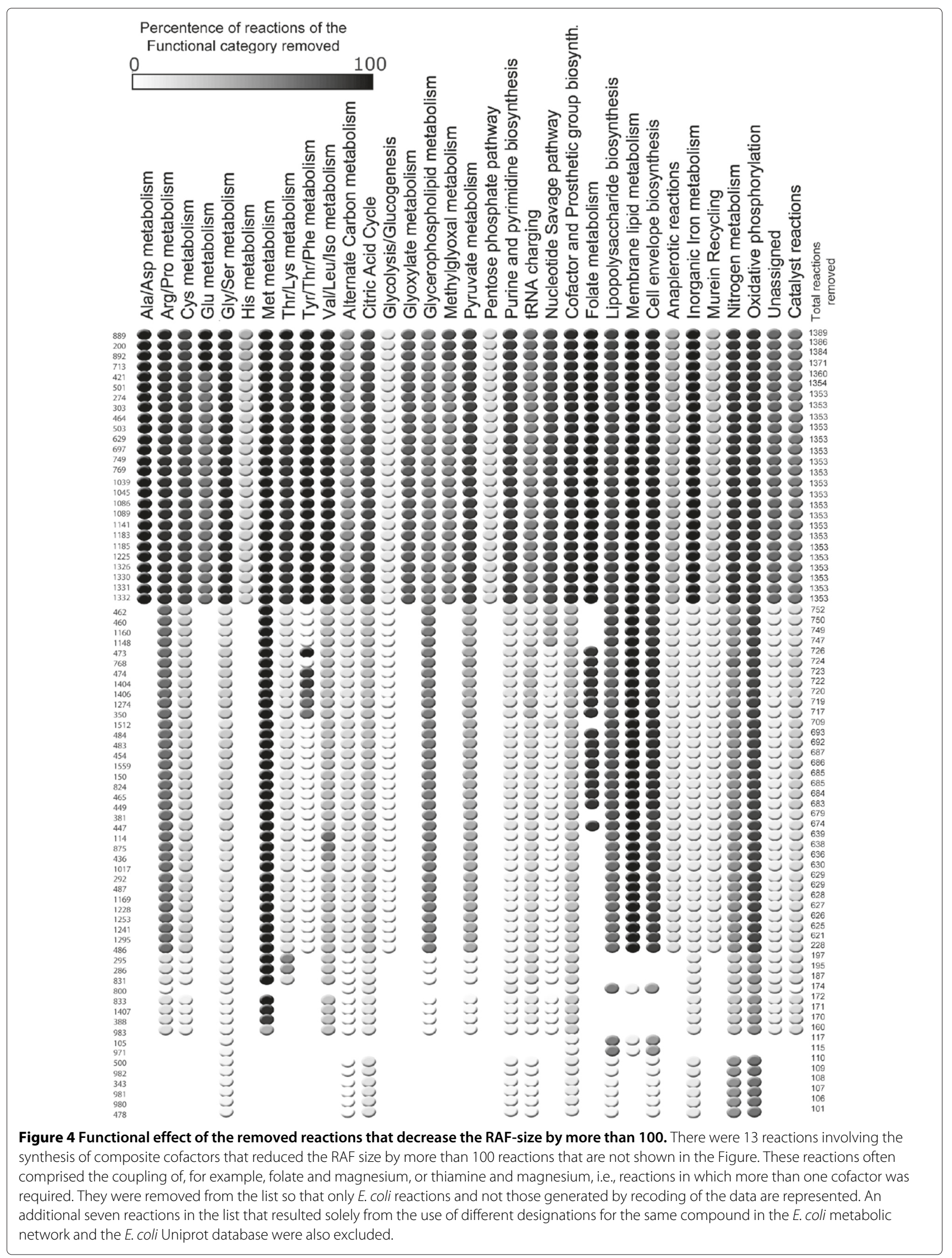


Table 6 Functional categories of the reactions affecting RAF size by more than 100 ("decr")

\begin{tabular}{|c|c|c|c|}
\hline ID & Functional annotation & Reaction & Decr \\
\hline 889 & Murein Recycling & LalaDgluMdap $+\mathrm{h}_{2} \mathrm{O} \rightarrow$ 26dap-M + LalaDglu & 1389 \\
\hline 200 & Murein Recycling & LalaDglu $\rightarrow$ LalaLglu & 1386 \\
\hline 892 & Murein Recycling & LalaLglu $+\mathrm{h}_{2} \mathrm{O} \rightarrow$ ala $-\mathrm{L}+$ glu- $\mathrm{L}$ & 1384 \\
\hline 713 & Glutamate Metabolism & atp + glu- $L+n h_{4} \rightarrow$ adp $+p i+h+g l n-L$ & 1371 \\
\hline 421 & Cofactor and Prosthetic Group Biosynthesis & ru $5 p-D \rightarrow d b 4 p+h+$ for & 1360 \\
\hline 501 & Cofactor and Prosthetic Group Biosynthesis & $\mathrm{g} 3 \mathrm{p}+\mathrm{h}+\mathrm{pyr} \rightarrow \mathrm{CO}_{2}+\mathrm{dxy} \mid 5 \mathrm{p}$ & 1354 \\
\hline 274 & Cofactor and Prosthetic Group Biosynthesis & $5 a p r u+h+$ nadph $\rightarrow$ 5aprbu + nadp & 1353 \\
\hline 303 & Alanine and Aspartate Metabolism & glu-L + oaa $\rightarrow$ akg + asp-L & 1353 \\
\hline 464 & Cofactor and Prosthetic Group Biosynthesis & $25 \mathrm{drapp}+\mathrm{h}+\mathrm{h}_{2} \mathrm{O} \rightarrow 5 \mathrm{apru}+\mathrm{nh}_{4}$ & 1353 \\
\hline 503 & Cofactor and Prosthetic Group Biosynthesis & $\mathrm{e} 4 \mathrm{p}+\mathrm{h}_{2} \mathrm{O}+\mathrm{nad} \rightarrow 4 \mathrm{per}+\mathrm{h}+\mathrm{h}+\mathrm{nadh}$ & 1353 \\
\hline 629 & Cofactor and Prosthetic Group Biosynthesis & $\mathrm{atp}+\mathrm{fmn}+\mathrm{h} \rightarrow \mathrm{fad}+\mathrm{ppi}$ & 1353 \\
\hline 697 & Nucleotide Salvage Pathway & $\mathrm{atp}+\mathrm{gmp} \rightarrow \mathrm{adp}+\mathrm{gdp}$ & 1353 \\
\hline 749 & Purine and Pyrimidine Biosynthesis & $a t p+g l n-L+h_{2} O+x m p \rightarrow a m p+p p i+h+h+g m p+g l u-L$ & 1353 \\
\hline 769 & Cofactor and Prosthetic Group Biosynthesis & $g t p+h_{2} \mathrm{O}+h_{2} \mathrm{O}+h_{2} \mathrm{O} \rightarrow 25 \mathrm{drapp}+$ ppi $+\mathrm{h}+\mathrm{h}+$ for & 1353 \\
\hline 1039 & Cofactor and Prosthetic Group Biosynthesis & $\operatorname{atp}+\operatorname{nad} \rightarrow \operatorname{adp}+$ nadp $+\mathrm{h}$ & 1353 \\
\hline 1045 & Cofactor and Prosthetic Group Biosynthesis & atp + dnad $+n h_{4} \rightarrow a m p+p p i+n a d+h$ & 1353 \\
\hline 1086 & Nucleotide Salvage Pathway & atp $+h+$ nicrnt $\rightarrow$ dnad + ppi & 1353 \\
\hline 1089 & Cofactor and Prosthetic Group Biosynthesis & $\mathrm{h}+\mathrm{h}+\mathrm{prpp}+\mathrm{quln} \rightarrow \mathrm{CO}_{2}+\mathrm{ppi}+$ nicrnt & 1353 \\
\hline 1141 & Cofactor and Prosthetic Group Biosynthesis & glu-L + ohpb $\rightarrow$ akg + phthr & 1353 \\
\hline 1183 & Cofactor and Prosthetic Group Biosynthesis & $\mathrm{dxyl} 5 \mathrm{p}+$ nad + phthr $\rightarrow \mathrm{CO}_{2}+\mathrm{pi}+\mathrm{pdx} 5 \mathrm{p}+$ nadh $+\mathrm{h}_{2} \mathrm{O}+\mathrm{h}_{2} \mathrm{O}+\mathrm{h}$ & 1353 \\
\hline 1185 & Cofactor and Prosthetic Group Biosynthesis & 4 per + nad $\rightarrow h+$ nadh + ohpb & 1353 \\
\hline 1225 & Cofactor and Prosthetic Group Biosynthesis & $5 \mathrm{aprbu}+\mathrm{h}_{2} \mathrm{O} \rightarrow 4 \mathrm{r} 5 \mathrm{au}+\mathrm{pi}$ & 1353 \\
\hline 1326 & Cofactor and Prosthetic Group Biosynthesis & dhap $+\mathrm{iasp} \rightarrow \mathrm{h}_{2} \mathrm{O}+$ quln $+\mathrm{pi}+\mathrm{h}_{2} \mathrm{O}$ & 1353 \\
\hline 1330 & Cofactor and Prosthetic Group Biosynthesis & $\mathrm{atp}+\mathrm{ribflv} \rightarrow \mathrm{adp}+\mathrm{h}+\mathrm{fmn}$ & 1353 \\
\hline 1331 & Cofactor and Prosthetic Group Biosynthesis & $4 \mathrm{r} 5 \mathrm{au}+\mathrm{db} 4 \mathrm{p} \rightarrow \mathrm{dmlz}+\mathrm{pi}+\mathrm{h}_{2} \mathrm{O}+\mathrm{h}_{2} \mathrm{O}$ & 1353 \\
\hline 1332 & Cofactor and Prosthetic Group Biosynthesis & $d m l z+d m l z \rightarrow 4 r 5 a u+r i b f l v$ & 1353 \\
\hline 462 & Purine and Pyrimidine Biosynthesis & cbasp $+\mathrm{h} \rightarrow$ dhor $-\mathrm{S}+\mathrm{h}_{2} \mathrm{O}$ & 752 \\
\hline 460 & Purine and Pyrimidine Biosynthesis & dhor-S + fum $\rightarrow$ orot + succ & 750 \\
\hline 1160 & Purine and Pyrimidine Biosynthesis & orot + prpp $\rightarrow$ orot $5 p+p p i$ & 749 \\
\hline 1148 & Purine and Pyrimidine Biosynthesis & $\mathrm{h}+\operatorname{orot} 5 \mathrm{p} \rightarrow \mathrm{CO}_{2}+\mathrm{ump}$ & 747 \\
\hline 473 & Tyrosine, Tryptophan, and Phenylalanine Metabolism & $2 d d a 7 p \rightarrow 3 d h q+p i$ & 726 \\
\hline 768 & Cofactor and Prosthetic Group Biosynthesis & $g t p+h_{2} \mathrm{O} \rightarrow$ ahdt $+\mathrm{h}+$ for & 724 \\
\hline 474 & Tyrosine, Tryptophan, and Phenylalanine Metabolism & $3 \mathrm{dhq} \rightarrow 3 \mathrm{dhsk}+\mathrm{h}_{2} \mathrm{O}$ & 723 \\
\hline 1404 & Tyrosine, Tryptophan, and Phenylalanine Metabolism & $3 \mathrm{dhsk}+\mathrm{h}+$ nadph $\rightarrow$ nadp + skm & 722 \\
\hline 1406 & Tyrosine, Tryptophan, and Phenylalanine Metabolism & $\operatorname{atp}+s k m \rightarrow \operatorname{adp}+s k m 5 p+h$ & 720 \\
\hline 1274 & Tyrosine, Tryptophan, and Phenylalanine Metabolism & pep + skm5p $\rightarrow 3 p s m e+p i$ & 719 \\
\hline 350 & Tyrosine, Tryptophan, and Phenylalanine Metabolism & $3 p s m e \rightarrow$ chor + pi & 717 \\
\hline 1512 & Nucleotide Salvage Pathway & $a t p+u m p \rightarrow a d p+u d p$ & 709 \\
\hline 484 & Cofactor and Prosthetic Group Biosynthesis & $a h d t+h_{2} \mathrm{O} \rightarrow d h p m p+p p i+h$ & 693 \\
\hline 483 & Cofactor and Prosthetic Group Biosynthesis & $\mathrm{dhpmp}+\mathrm{h}_{2} \mathrm{O} \rightarrow \mathrm{dhnpt}+\mathrm{pi}$ & 692 \\
\hline 454 & Cofactor and Prosthetic Group Biosynthesis & dhnpt $\rightarrow 6$ hmhpt + gcald & 687 \\
\hline 1559 & Cofactor and Prosthetic Group Biosynthesis & chor + gln- $\mathrm{L} \rightarrow$ 4adcho + glu- $\mathrm{L}$ & 686 \\
\hline 150 & Cofactor and Prosthetic Group Biosynthesis & 4 adcho $\rightarrow 4 a b z+p y r+h$ & 685 \\
\hline 824 & Cofactor and Prosthetic Group Biosynthesis & $6 \mathrm{hmhpt}+$ atp $\rightarrow 6 \mathrm{hmhptpp}+\mathrm{h}+\mathrm{amp}$ & 685 \\
\hline
\end{tabular}


Table 6 Functional categories of the reactions affecting RAF size by more than $\mathbf{1 0 0}$ ("decr") (Continued)

\begin{tabular}{|c|c|c|c|}
\hline 465 & Cofactor and Prosthetic Group Biosynthesis & $4 a b z+6 h m h p t p p \rightarrow d h p t+p p i$ & 684 \\
\hline 449 & Cofactor and Prosthetic Group Biosynthesis & $a t p+d h p t+g l u-L \rightarrow a d p+p i+h+d h f$ & 683 \\
\hline 381 & Purine and Pyrimidine Biosynthesis & atp + gln-L $+h_{2} O+u t p \rightarrow a d p+p i+h+h+$ glu-L + ctp & 679 \\
\hline 447 & Cofactor and Prosthetic Group Biosynthesis & $\mathrm{dhf}+\mathrm{h}+$ nadph $\rightarrow$ nadp + thf & 674 \\
\hline 114 & Valine, Leucine, and Isoleucine Metabolism & $\mathrm{h}+\mathrm{pyr}+\mathrm{pyr} \rightarrow$ alac-S $+\mathrm{CO}_{2}$ & 639 \\
\hline 875 & Valine, Leucine, and Isoleucine Metabolism & alac-S $+h+$ nadph $\rightarrow 23 \mathrm{dhmb}+$ nadp & 638 \\
\hline 436 & Valine, Leucine, and Isoleucine Metabolism & $23 \mathrm{dhmb} \rightarrow 3 \mathrm{mob}+\mathrm{h}_{2} \mathrm{O}$ & 636 \\
\hline 1017 & Cofactor and Prosthetic Group Biosynthesis & $3 \mathrm{mob}+\mathrm{h}_{2} \mathrm{O}+$ mlthf $\rightarrow 2 \mathrm{dhp}+$ thf & 630 \\
\hline 292 & Cofactor and Prosthetic Group Biosynthesis & asp- $\mathrm{L}+\mathrm{h} \rightarrow$ ala- $\mathrm{B}+\mathrm{CO}_{2}$ & 629 \\
\hline 487 & Cofactor and Prosthetic Group Biosynthesis & $2 \mathrm{dhp}+\mathrm{h}+$ nadph $\rightarrow$ nadp + pant $-\mathrm{R}$ & 629 \\
\hline 1169 & Cofactor and Prosthetic Group Biosynthesis & ala-B + atp + pant $-R \rightarrow a m p+p p i+$ pnto- $R+h$ & 628 \\
\hline 1228 & Cofactor and Prosthetic Group Biosynthesis & atp + pnto- $R \rightarrow 4$ ppan $+h+$ adp & 627 \\
\hline 1253 & Cofactor and Prosthetic Group Biosynthesis & 4 ppan + ctp + cys-L $\rightarrow 4$ ppcys + ppi $+h+$ cmp & 626 \\
\hline 1241 & Cofactor and Prosthetic Group Biosynthesis & 4 ppcys $+h \rightarrow \mathrm{CO}_{2}+$ pan $4 p$ & 625 \\
\hline 1295 & Cofactor and Prosthetic Group Biosynthesis & atp $+h+$ pan $4 p \rightarrow$ dpcoa + ppi & 621 \\
\hline 486 & Cofactor and Prosthetic Group Biosynthesis & $\mathrm{atp}+\mathrm{dpcoa} \rightarrow \mathrm{adp}+\mathrm{h}+\mathrm{coa}$ & 619 \\
\hline 295 & Threonine and Lysine Metabolism & $a s p-L+a t p \rightarrow 4 p a s p+a d p$ & 197 \\
\hline 286 & Threonine and Lysine Metabolism & 4 pasp $+h+$ nadph $\rightarrow$ aspsa + nadp + pi & 195 \\
\hline 831 & Threonine and Lysine Metabolism & aspsa $+h+$ nadph $\rightarrow$ hom $-L+$ nadp & 187 \\
\hline 800 & Unassigned & $\mathrm{CO}_{2}+\mathrm{h}_{2} \mathrm{O} \rightarrow \mathrm{h}+\mathrm{hCO}_{3}$ & 174 \\
\hline 833 & Methionine Metabolism & hom-L + succoa $\rightarrow$ coa + suchms & 172 \\
\hline 1407 & Methionine Metabolism & cys- $L+$ suchms $\rightarrow$ cyst- $L+$ succ + h & 171 \\
\hline 388 & Methionine Metabolism & cyst- $L+h_{2} \mathrm{O} \rightarrow$ hcys-L + pyr $+\mathrm{nh}_{4}$ & 170 \\
\hline 983 & Methionine Metabolism & $a t p+h_{2} \mathrm{O}+$ met- $\mathrm{L} \rightarrow$ amet + ppi + pi & 160 \\
\hline 105 & Membrane Lipid Metabolism & $\mathrm{accoa}+\mathrm{atp}+\mathrm{hCO}_{3} \rightarrow \mathrm{adp}+\mathrm{pi}+$ malcoa $+\mathrm{h}$ & 117 \\
\hline 971 & Membrane Lipid Metabolism & $\mathrm{ACP}+\mathrm{malcoa} \rightarrow \mathrm{COa}+\mathrm{malACP}$ & 115 \\
\hline 500 & Cofactor and Prosthetic Group Biosynthesis & $d x y l 5 p+h+$ nadph $\rightarrow 2$ me4p + nadp & 110 \\
\hline 982 & Cofactor and Prosthetic Group Biosynthesis & $2 m e 4 p+c t p+h \rightarrow 4 c 2 m e+p p i$ & 109 \\
\hline 343 & Cofactor and Prosthetic Group Biosynthesis & $4 c 2 m e+a t p \rightarrow 2 p 4 c 2 m e+h+a d p$ & 108 \\
\hline 981 & Cofactor and Prosthetic Group Biosynthesis & $2 p 4 c 2 m e \rightarrow 2 m e c d p+c m p$ & 107 \\
\hline 980 & Cofactor and Prosthetic Group Biosynthesis & $2 m e c d p+f|x r+f| x r+h \rightarrow f l \times s O+h_{2} O+h 2 m b 4 p+f l x s o$ & 106 \\
\hline 478 & Cofactor and Prosthetic Group Biosynthesis & $d m p p+i p d p \rightarrow$ grdp + ppi & 101 \\
\hline
\end{tabular}

Chemical species are symbolic represented.

size by more than 100 reactions, the functional categories affected are given in Figure 4. Table 6 lists the actual reactions themselves, including their function category. The same pattern shows up for multiple repetitions of the algorithm. However, for readability of Figure 3 we have only shown the results of ten such runs.

The reactions whose removal reduces the size of the RAF the most involve nitrogen assimilation, glutamine synthase (GS) and mureine recycling. The latter seems surprising but is easily explained, because in the E. coli network we use here [12], a mureine degradation product (anhgm4p, N-acetyl-D-glucosamine $\mathrm{N}$-acetylmuramyl-tetrapeptide), originally a periplasmic product but transferred to the food set (see Experimental), is one of the food sources of glutamate, the substrate for ammonium incorporation in the GS reaction. Within this RAF network, the highly dependent cofactor biosynthesis network is initially only operational at the expenses of glutamate formation from the mureine degradation pathway. If glutamate (or GS) is removed, no nitrogen can be incorporated and the reaction network stalls at many reactions, much like the case of ATP above. Glutamine synthase is the entry point of nitrogen in E. coli metabolism [53], and without nitrogen, no cofactors, amino acids or bases can be generated, so this result makes sense. 
Also of interest is the central role that cofactor biosynthesis assumes in the $E$. coli RAF. Of the 76 reactions that reduce the size by more that 100 reactions, 43 (57\%) fall in the functional category synthesis of cofactor and prosthetic groups. This is intuitively understandable because cofactors are catalysts. It furthermore underscores the importance of cofactors as mediators of metabolism $[11,54,55]$. Following the cofactors in terms of effect on the RAF when removed, are amino acids (21), nucleotide biosynthesis (9), and three others.

Finally, there are clear intermediate levels in the (reducing) RAF sets. As Figure 3 shows, there are significant drops in RAF size first to around 1100 reactions and then to around 400 reactions, which occur in almost all of the 10 runs. Interestingly, all these (roughly) size- 400 subsets consist primarily of reactions from the 434-reaction CAF subset, indicating that this is kinf of "robust" core of the maxRAF and furthermore indicating the existence of modularity in the RAF set.

\section{The effects of removing molecules}

The irrRAF search algorithm gives insight into the importance of individual reactions on the size of the RAF set. We can perform a similar procedure for the molecules. Figure 5 shows the reduction in the size of the maxRAF set (in number of reactions) when an individual molecule type is removed from the network (its "importance"), against the number of reactions this molecule is involved in either as a reactant, product, or catalyst (its "involvement"), for all 1199 molecule types. Note that, as with removing reactions, there is a clear separation into a few different levels of RAF sizes when removing molecules.

Obviously the molecules that are involved in many reactions (the upper set of points in Figure 5) have a large importance, that is, they reduce the RAF set by more than 1300 reactions when removed. There are 76 compounds in this group, including all of the cofactors except folate and coenzyme A, many amino acids and core carbon coumponds like ribose-5-phosphate or phosphoenolpyruvate (Table 6). Removal of such compounds should have a large effect. However, there are also many molecules that have a very low involvement (less than 50 reactions, except coenzyme A) but a very high importance in that they reduce the RAF set by more than 600 reactions when removed. There are 52 compounds in this class, including folate, but mostly central metabolites of core biosynthesis. The third group of molecules encompasses 1071 metabolites with both low involvement and low importance. These might be considered as peripheral in E. coli metabolism. But if this is the periphery, it would leave only 128 compounds in the core. This might seem like an unrealistically small core, but we note that there are only 303 essential genes in E. coli and the metabolic network model of $E$. coli needs just 250 genes to be able to produce biomass [47] (see Conclusions).

After protons ("h" in Figure 5) and the generic catalyst "Protein", the most frequent participant in an $E$. coli reaction is water. The involvement of water in many

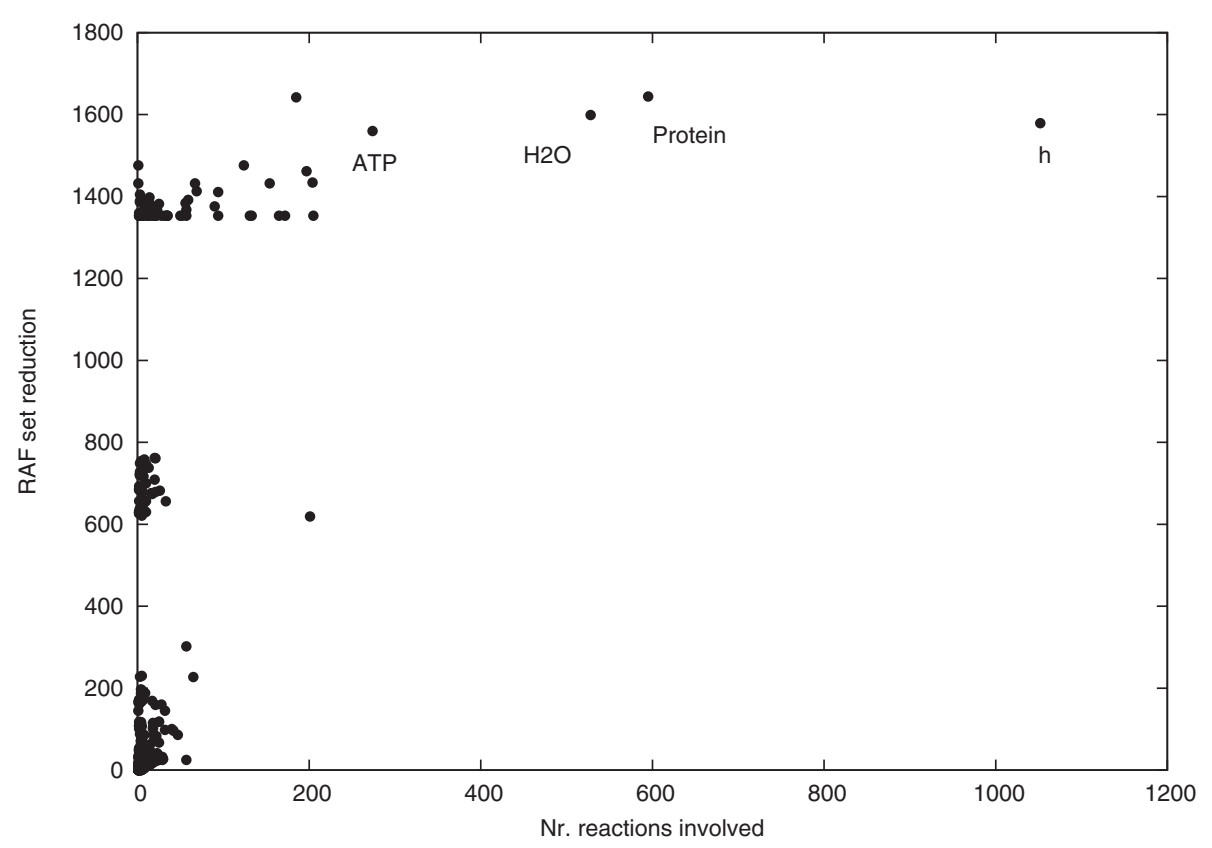

Figure 5 Involvement and importance of molecule types. Relationship between the removal of each molecule and the number of reaction where it participates. Each dot corresponds to a different molecule. 
reactions might seem trivial, but water is more central to metabolism than one might think: $70 \%$ of the water molecules in the cytosol of exponentially growing aerobic E. coli cultures do not stem from the aqueous medium, but they are synthesized through E. coli metabolism [56].

\section{Can real food sets support the RAF structure?}

If we set the food set to biologically realistic conditions where $E$. coli has been shown to grow, the RAF network collapses and less than $10 \%$ of the reactions are retrieved (Table 7). This apparently negative result shows that the introduction of an additional level of regulation (a reaction only occurs if the reaction catalyst is present in the network) has a massive impact in the way current cells function. As observed for the RAF network with a food set of 123 molecules, the catalysts have a different impact in the size of the RAF (Additional file 1: Table S1). The next step is to check what needs to be further introduced in this reduced food set in order for the essential metabolic pathways such as pentose metabolism, glycolysis, citric cycle, amino acid and cofactor biosynthesis to become functional. While finding the minimal food set is an NPcomplete problem, finding a food set that creates a CAF network comprising those metabolic pathways is possible. In fact, besides the addition of the obligate autocatalytic metabolite ATP, using the glucose-6-phosphate food set with the addition of just seven catalysts (NAD, FAD, PLP, thiamin, CoA, lipoate and some cobinamine form), a CAF network with the same size of the resulting RAF set and containing 1517 reactions is retrieved. Although with this food set only $85 \%$ of the initial network is retrieved, the main E. coli cytosolic metabolism is still captured (Table 7). Interestingly, the autocatalytic metabolites that Szathmary and coworkers found for the E.coli metabolic network grown in minimum media also included CoA, NAD and ATP [11]. The difference between their study and ours is that we also took into consideration the cofactor dependency of the enzyme catalyzing the reactions. Thus, our list includes FAD, PLP and thiamin as autocatalytic metabolites due to the existence of several enzymes that are dependent on these cofactors, it includes cobalamin that $E$. coli naturally uptakes from the environment and also lipoate, whose synthesis is not described within the network.

However, not all of these 39 food molecules are essential to maintain a similar RAF size (Table 7) and this solution is not unique. For instance, the removal of either $\mathrm{SO}_{4}$ or $\mathrm{S}_{2} \mathrm{O}_{3}$ has no effect on the size of either the RAF or the CAF because the presence of one can sustain $E$. coli's growth by providing a sulfur source. A more interesting result comes from the removal of glucose-6-phosphate (the only obvious carbon source) from the food set, since its removal also does not affect the size of the RAF. In this case, the source of carbon becomes ATP itself, by
Table 7 Impact of removal of molecules from the "real" $E$. coli food set in the RAF and CAF size

\begin{tabular}{|c|c|c|}
\hline Food molecule & RAF size decrease & CAF size decrease \\
\hline $\mathrm{Ca}^{2+}$ & 7 & 7 \\
\hline $\mathrm{Cl}$ & 2 & 2 \\
\hline $\mathrm{CO}^{2+}$ & 33 & 33 \\
\hline $\mathrm{Cu}^{2+}$ & 27 & 27 \\
\hline$k^{+}$ & 155 & 360 \\
\hline $\mathrm{Mg}^{2+}$ & 1117 & 1117 \\
\hline $\mathrm{Mn}^{2+}$ & 133 & 133 \\
\hline $\mathrm{MoO}_{4}^{2-}$ & 24 & 24 \\
\hline $\mathrm{Na}^{+}$ & 3 & 3 \\
\hline $\mathrm{Ni}^{2+}$ & 11 & 11 \\
\hline $\mathrm{NO}_{3}$ & 3 & 3 \\
\hline $\mathrm{SeO}_{4}^{2-}$ & 6 & 6 \\
\hline TrimethylamineN - oxide & 2 & 2 \\
\hline $\mathrm{S}_{2} \mathrm{O}_{3}^{2-}$ & 0 & 0 \\
\hline $\mathrm{WO}_{4}^{2-}$ & 11 & 11 \\
\hline $\mathrm{Zn}^{2+}$ & 631 & 631 \\
\hline ATP & 1206 & 1206 \\
\hline $\mathrm{O}_{2}$ & 47 & 47 \\
\hline genCat & 492 & 492 \\
\hline Protein & 1352 & 1352 \\
\hline RNA & 88 & 88 \\
\hline spont & 41 & 41 \\
\hline$x$ & 1447 & 1447 \\
\hline Glutathione & 30 & 30 \\
\hline $\mathrm{H}_{2} \mathrm{O}$ & 0 & 0 \\
\hline $\mathrm{Fe}^{2+}$ & 91 & 91 \\
\hline $\mathrm{NH}_{4}$ & 0 & 0 \\
\hline $\mathrm{H}_{3} \mathrm{PO}_{4}$ & 0 & 0 \\
\hline $\mathrm{SO}_{4}$ & 0 & 0 \\
\hline Iron - Sulfur - cluster & 0 & 866 \\
\hline$N A D *$ & 1261 & 1261 \\
\hline FAD* & 0 & 1162 \\
\hline Glucose - 6 - phosphate & 0 & 0 \\
\hline Pyridoxal5' - phosphate* & 0 & 673 \\
\hline $\mathrm{COA} *$ & 1099 & 1099 \\
\hline $\mathrm{H}_{2} \mathrm{~S}$ & 0 & 194 \\
\hline Thiamin* & 2 & 417 \\
\hline Adenosylcobinamide* & 10 & 10 \\
\hline Lipoate* & 8 & 8 \\
\hline
\end{tabular}

"indicates the 7 added molecules.

its conversion into adenosine in the nucleotide salvage pathway. This captures an aspect of E. coli's versatility since $E$. coli can grow aerobically or anaerobically, both in 
silico and in vivo, using adenosine as the sole carbon and nitrogen source [57].

To check the modularity of this CAF network in terms of the metabolic pathways organization, we determined the hierarchical levels of each reaction, grouped by $E$. coli KEGG metabolic pathways. Based on the relationship between the different $E$. coli metabolic pathways with the 53 hierarchical levels retrieved, it is possible to represent the CAF network in a tree-based structure containing several grouped pathways (Figure 6). In this representation,

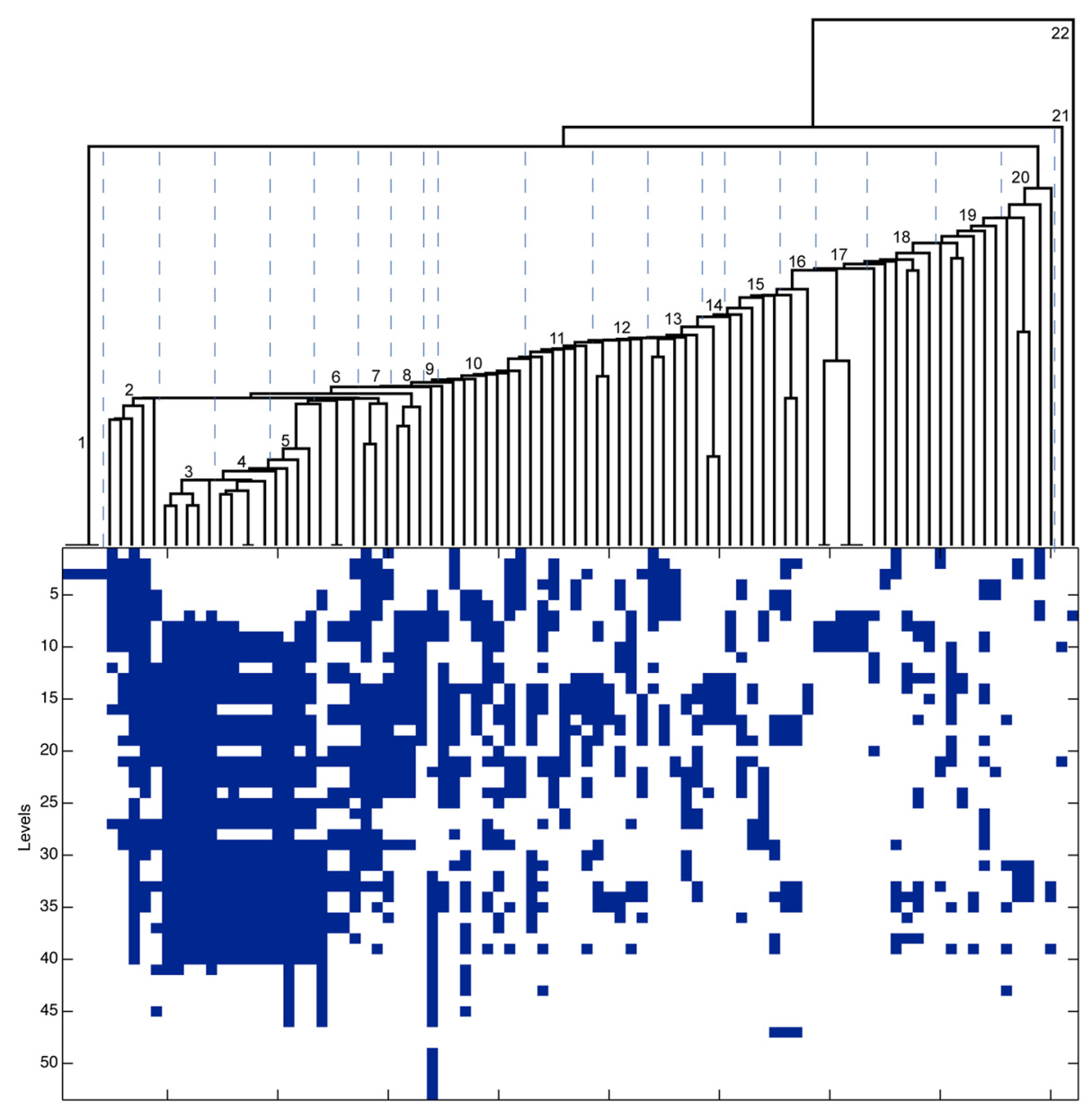

Figure 6 Hierarchical levels of E. coli CAF network using a "real"? Food set. Top- Hierarchical clustering dendrogram of E. coli metabolic pathways (leafs) according to the hierarchical levels defined in the text. Bottom- Heatmap representing the occurrence of reactions from each one of metabolic pathways (columns) and the 53 hierarchical levels of the CAF network (rows). Blue squares represent the occurrence of at least one reaction from that pathway in a given level. Numbers represent grouped pathways. 1- Nucleotide-excision-, mismatch-, and base-excision- repair; DNA-replication. 2- Glycolysis/gluconeogenesis; methane-, carbon-, amino acids biosynthesis, glycine, serine and threonine- metabolism. 3- Fatty acid biosynthesis and metabolism; valine, leucine and isoleucine-, geraniol- and fatty-acid- degradation. 4 - Lysine degradation; tryptophan metabolism; limonene-, caprolactam- degradation; beta-alanine metabolism. 5- Unsaturated fatty-acids biosynthesis, biotin-, propanoate- and butanoate- metabolism. 6- Glycerophospholipid- and alpha-linolenic acid metabolism; ethylbenzene degradation; pantothenate and CoA biosynthesis. 7- Purine-, pyrimidine- and porphyrin- metabolism; Valine, leucine and isoleucine biosynthesis. 8- Oxocarboxylic acid metabolism; phenylalanine, tyrosine and tryptophan biosynthesis. 9- Lipopolysaccharide biosynthesis. 10- Arginine and proline-, amino sugar and nucleotide sugar-, glycerolipid-, histidine-, glyoxylate- and dicarboxylate metabolism; benzoate degradation; nicotinate-, starch and sucrose metabolism. 11- Quinone biosynthesis; pyruvate-, galactose- metabolism; lysine biosynthesis; PLP metabolism; aminoacyl-tRNA biosynthesis. 12-Cysteine and methionine metabolism; siderophore-group nonribosomal-peptides biosynthesis; glutathione-, citrate-cycle, sulfur- metabolism. 13- Pentose phosphate pathway; fructose-, mannose metabolism; pentose and glucuronate interconversions; peptidoglycan- and folate- biosynthesis. 14- Phenylalanine metabolism; novobiocin biosynthesis. 15- Tyrosine-, riboflavin and cyanoamino-acid metabolism; terpenoid-backbone biosynthesis; Selenocompound metabolism. 16- Thiamine-, Sulfur-relay system, D-Alanine metabolism. 17- Dioxin-, Xylene-, Chloroalkane-, naphthalene-, aromatic- degradation. 18- C5-Branched dibasic acid-, inositolphosphate-, oxidative phosphorylation, nitrogen-, two-component system, taurinemetabolism. 19- lipoic acid-, alanine, aspartate, glutamate-, D-glutamine and D-glutamate- metabolism; nitrotoluene degradation; folate one-carbon pool; ascorbate metabolism. 20- Aminobenzoate degradation; streptomycin-, polyketide-sugar biosynthesis; RNA-, toluene- degradation. 21- Arachidonic acid metabolism. 22- phosphotransferase system. 
thr reaction network is organized into 53 different levels of iteration, starting from the 39-molecule food set. Similar patterns of occurrence with increasing distance from the food set indicate that the molecules and catalysts necessary for each of their reactions arise at the same iteration. The synthesis of lipopolysaccharide, the major component of E. coli outer membrane (group 9 in Figure 6) is the last process to be concluded suggesting a high dependency of lipopolysaccharide biosynthesis on other metabolic pathways and, consequently, its late emergence during metabolic evolution.

\section{Conclusions}

Although autocatalytic networks are found in biological systems, the systematic impact of including the metal and cofactor protein dependencies as catalysts in metabolic networks under the RAF context has not been previously investigated. The present analyses shows that, within this framework, the E. coli metabolic network can indeed be expressed as an RAF set. RAFs also recover the modularity and hierarchical behavior of the $E$. coli metabolic network - in particular they underscore the crucial role of cofactors as the prime mediators of metabolism, a recurring theme in the study of metabolic architecture $[11,55,58]$. Here we have shown the important role of metals and molecules such as NAD, ATP and CoA in breaking autocatalytic cycles and sustaining the network complexity. This result is in agreement with findings of Heinrich and coworkers [59], who analysed the scopes of compounds and expansion within KEGG metabolic networks and showed the crucial role of the inclusion of these metabolites in the expansion and robustness increase of metabolic networks. Moreover, by also including the metal and cofactor dependencies of the proteins, we extended this set of compounds by identifying additional molecules such as thiamin and PLP as autocatalytic metabolites within E. coli metabolism. Thus, RAFs can clearly be used to explore the biological importance of molecules/catalyst within a cell and their interrelationships. But there are caveats.

Among the caveats to the present analyses, the underlying databases are not complete. As one example, biotin does not occur as a cofactor in the present annotations from the UNIPROT database for E. coli, but it is known to generally be required in ATP-dependent carboxylation reactions, for example in that catalyzed by acetyl-CoA carboxyase [60]. Another caveat is that RAFs exclude, by definition, a potentially important kind of reaction, namely spontaneous reactions that have no catalysts at all. There are a number of important reactions in biology that are spontaneous. A prime example is the first step in $\mathrm{CO}_{2}$ assimilation in methanogens, which involves the spontaneous (non-enzymatic) formation of $\mathrm{N}$-carboxymethanofuran [61]. In modern environments, about a billion tons of carbon are processed via this spontaneous reaction each year [62] and spontaneous reactions of this type might have been important in early evolution [55]. For this reason we introduced the catalyst "spont" for reactions that are annotated as spontaneous, of which there are 17 in the present analysis (Table 4).

Another caveat is promiscuity (or messiness) in enzyme function, that is, the inherent ability of enzymes to catalyze several different selectable reactions [63], whereby usually only one function appears on metabolic maps. This opens the possibility to have additional parallel reactions catalyzed by different cofactors within the metabolic network. Early in the evolution of enzymes, that is at the dawn of protein folds and enzyme families, catalytic specificity was probably rare. In modern $E$. coli, the full extent to which gene products can substitute for each other is not known, although in one classical study, 620 genes in $E$. coli were found to be essential in rich medium (263 of which had no known function), while 3126 were dispensable [64]. A more recent study found that only 303 E. coli genes were essential (37 of which had no known function), and 3985 were dispensable [65]. This indicates that there is a great deal of redundancy and/or environmental specificity [66] built into E. coli metabolism and that there is still much to be learned about its map.

Finally, although we can easily find RAFs, including CAFs and irreducible RAFs, there are also limits to what can be calculated. For example, we have shown here that finding the minimal food set needed to maintain a given $\mathrm{RAF}$ is a computationally intractable (NP-complete) problem in general. So too is finding a smallest irrRAF [35], but here this seems not interesting as the smallest irrRAF turn out to be of size one (so-called "trivial" irrRAFs). Instead, it would be of more interest to find a largest irrRAF within the E. coli metabolism network. However, at present it is not clear whether this can be computed efficiently.

\section{Do these findings bear upon early chemical evolution?}

The origin and initial interest in RAFs stem from early speculation about chemical evolution [4] and the possibility that autocatalytic sets might have played a role as a means of self organization en route to higher complexity prior to the advent of genetically specified catalysts. One prerequisite for the existence of RAFs in the real world is of course a set of food molecules provided by the environment. Another prerequisite is that the laws of thermodynamics must be obeyed, thus that the overall reaction needs to release energy. Amend et al. [45] have examined these two properties in the context of hydrothermal vents, where organic synthesis from smaller "food" building blocks is thermodynamically favored, owing to the exergonic nature of the interactions between $\mathrm{H}_{2}$ and $\mathrm{CO}_{2}$ to yield organic products. 
Of interest, Kauffman's speculations entailed the synthesis of large peptides from small ones, and early work showed that the synthesis of both amino acids and peptides under hydrothermal vent conditions are exergonic processes [67], whereby a typical microbe is more than $50 \%$ protein by weight $[68,69]$.

$E$. coli is a heterotroph that can live anaerobically but can also, like human mitochondria, use $\mathrm{O}_{2}$ as the terminal electron acceptor in its ATP-generating electron transport chain. In that sense the $E$. coli metabolic network is hardly an ideal model for early chemical evolution. In addition, during evolution abiotic catalysts and metals in primordial RAF sets have been replaced by sophisticated chemical catalysts, as studies of metal and cofactor gains and losses across protein families have shown [70-72]. Comparative genomic analysis of the distribution of trace elements in current genomes indicate that the loss of a metal or cofactor is more frequent that their respective gain [73]. Moreover, phylogenomic analysis of protein structures also concluded that Fe, Mn, and Mo were preferentially selected by early life forms and were replaced or lost during evolution [74], such that in early chemical evolution, metal dependency was probably higher. Moreover, throughout evolution, protein have often been replaced by analogous proteins of similar or identical functions. The presence across genomes of metal-independent (class I and Ia) and metal-dependent (class II) aldolases is just one example [75-77] where, possibly due to later sugar metabolism adaptations, these enzymes likely replaced an ancestral bifunctional fructose 1,6-bisphosphate aldolase/phosphatase enzyme involved in gluconeogenis [78].

Finally, early enzymes probably had a more relaxed substrate specificity than in modern metabolism. This reasoning is the basis of "The Game of the Pentose Phosphate Cycle" study where Meleindez-Hevia and Isidoro showed that generic aldolases and ketolases could generate a large set of sugar phosphate interconversions and the subsequent growing specificity of the enzymes lead to a minimal solution, that in fact is equivalent to the naturally occurring pathway in $E$. coli to recycle pentoses to hexoses [79]. Similarly, Noor et al expanded contemporary central carbon metabolism by assuming a relaxed specificity of enzymes [80]. With this methodology, they showed that the central carbon metabolism in $E$. coli connects input sugars and the key precursors metabolites essential for biomass and energy production by the minimal number of enzymes, suggesting that contemporary metabolism is a small subset of the original possibilities.

The metabolic network of $E$. coli represents the result of billions of years of catalytic refinement through natural variation and natural selection. However some of the properties germane to early evolution are common to all life forms and should still be at least partially conserved and, it is generally of interest to know whether the beststudied metabolic system is an RAF. With a few restrictions, for example the introduction of generic catalysts where no cofactors are involved, it indeed is. This is an encouraging result for future studies on the metabolic networks of ostensibly more primitive organisms such as acetogens and methanogens [44,81] whose carbon and energy metabolism is not only simpler than that of $E$. coli, but also more similar to chemistry at hydrothermal vents, and whose metabolism furthermore involves a more prominent role of catalysis by metals $[46,82]$.

The critical role of cofactors in the E. coli RAFs might point to an interesting aspect of early chemical evolution. We see here that the size, hence in some respects the complexity, of RAFs within the E. coli metabolic network are dependent upon cofactors: a small number of catalysts that promote a large number of reactions each. Regardless of where life arose, in the very earliest phases of chemical evolution, there must have been both thermodynamically controlled reactions (the most stable compounds accumulate) and kinetically controlled reactions (the most rapidly synthesized products accumulate). By lowering the activation energy of a reaction, cofactors influence the latter class of reactions more than the former as seen in the PLP example before mentioned [20]. Hence the spontaneous, and perhaps inorganically catalyzed, synthesis of small amounts of a small number of organic cofactors at the onset of chemical evolution could have strongly influenced the nature of compounds that subsequently accumulated. With the advent of proteins, this principle might not have been discarded, depending on whether one interprets the $E$. coli metabolic map as harboring some relics from early evolution, or not. In our opinion, some of these original imprints may still be present in the metabolism of modern organisms, as seen by their recurrent use of the same set of metals and organic cofactors as catalysts of biologic reactions, a set much smaller than the number of protein families that have evolved to catalyze them. The directives of the continuity principle in evolution demand that complex biochemistries had to be preceded by simpler chemistries. Thus, the initial RAF set would certainly be much simpler than the one analyzed in this paper but migth have already manifest the principle of cofactor and metal dependencies as recurrently is observed across studies showing their central role in modern metabolism $[11,59,83]$.

We have shown that the $E$. coli reaction network can produce useful insights into primordial RAFs by identifying the essential role of metals and molecules such as ATP, CoA or thiamin in metabolism. This suggests the existence of some sort of abiotic autocatalysis at the onset of primordial metabolic networks. We have also shown here that maximal RAFs can be efficiently detected in real biological data, but the identification of large irreducible 
RAFs and the minimal food sets required to support a maximal RAF remain challenging problems.

\section{Appendix \\ Proof that min-F RAF and min-F Generation are NP-complete}

First note that both problems are in the complexity class $\mathrm{NP}$, since we can determine in polynomial time (in the size of the input) whether a set of reactions is $F^{\prime}$-generated and/or an RAF, for any given set $F^{\prime}$.

Next, notice that it suffices to show that the simpler problem 'min-F generation' is NP-complete, since for any instance $I$ of 'min-F generation' there is a corresponding instance $I^{\prime}$ of 'min-F RAF' obtained by (i) making every molecule in $X$ catalyze every reaction in $\mathcal{R}$, and (ii) taking $\mathcal{R}=\mathcal{R}^{\prime}$ and $X$ to be the molecules in the support of $\mathcal{R}^{\prime}$; under this correspondence, $I^{\prime}$ has an affirmative answer if and only if $I$ does.

We will show that 'min-F generation' is NP-complete by exhibiting a (polynomial-time) reduction from the following set-theoretic decision problem.

\section{Exact cover by 3-sets (X3C)}

INSTANCE: Finite set $Y$ with $|Y|=3 q, q$ an integer; collection $S$ of 3-element subset of $X$.

QUESTION: Does $S$ contain an exact cover for $Y$, i.e., a subcollection $S^{\prime}$ of $S$ such that every element of $Y$ occurs in exactly one member of $S^{\prime}$ ?

The decision problem $\mathrm{X} 3 \mathrm{C}$ was one of the early ones to be shown NP-complete by Karp [84]. We may assume, without loss of generality, that in X3C every element of $Y$ occurs in at least one element (3-element subset) of $S$. As an example of $\mathrm{X} 3 \mathrm{C}$, consider the set $Y=\{a, b, c, d, e, f\}$ and

$$
S=\{\{a, b, c\},\{a, d, e\},\{d, e, f\}\} .
$$

In this case $S^{\prime}=\{\{a, b, c\},\{d, e, f\}\}$ is the (unique) subset of $S$ that provides an exact 3-cover for $Y$.

Given an arbitrary instance $(Y, S)$ of $\mathrm{X} 3 \mathrm{C}$, we will construct an associated set $\mathcal{R}_{(Y, S)}$ of reactions. The set of molecules $X$ involved in these reactions is the (disjoint) union $Y \cup S \cup W$, where $W:=\left\{w_{s}: s \in S\right\}$. Thus $|X|=|Y|+|S|+|W|=3 q+2|S|$. Moreover, we will take the food set $F$ to consist of all of $X$.

We next describe the reactions. First impose an arbitrary total order $<$ on $Y$. Then for each set $s=\{a, b, c\} \in$ $S$, with $a<b<c$, consider the set $\mathcal{R}_{s}$ consisting of the following three reactions:

$$
\begin{aligned}
& s \rightarrow a+b+c \\
& a+b \rightarrow w_{s} \\
& w_{s}+c \rightarrow s
\end{aligned}
$$

Then define $\mathcal{R}_{(Y, S)}:=\bigcup_{s \in S} \mathcal{R}_{S}$, and observe that $\mathcal{R}_{(Y, S)}$ is $F$-generated, and that $\left|\mathcal{R}_{(Y, S)}\right|=3|S|$.

In the example above, with $s=\{a, b, c\}, s^{\prime}=\{a, d, e\}$ and $s^{\prime \prime}=\{d, e, f\}$, and with the molecules ordered alphabetically, $\mathcal{R}_{(Y, S)}$ comprises the nine reactions:

$$
\begin{aligned}
& s \rightarrow a+b+c, a+b \rightarrow w_{s}, w_{s}+c \rightarrow s ; \\
& s^{\prime} \rightarrow a+d+e, a+d \rightarrow w_{s^{\prime}}, w_{s^{\prime}}+e \rightarrow s^{\prime} ; \\
& s^{\prime \prime} \rightarrow d+e+f, d+e \rightarrow w_{s^{\prime \prime}}, w_{s^{\prime \prime}}+f \rightarrow s^{\prime \prime} .
\end{aligned}
$$

Claim: $(Y, S)$ has an exact 3-cover, if and only if $\mathcal{R}_{(Y, S)}$ is $F^{\prime}$-generated for some subset $F^{\prime}$ of $F$ of size at most $q=|Y|$.

Proof of Claim. First, suppose that $S^{\prime}$ is an exact 3-cover for $Y$. Then $\left|S^{\prime}\right|=q$ and every element of $Y$ is generated by a reaction that has its (sole) reactant in $S^{\prime}$. It follows that for every $s \in S$ (say, $s=\left\{y, y^{\prime}, y^{\prime \prime}\right\}$ with $y<y^{\prime}<y^{\prime \prime}$ ) the associated reaction $y+y^{\prime} \rightarrow w_{s}$ has reactants that can be generated from $S^{\prime}$. This ensures, in turn, that the other associated reaction $w_{s}+y^{\prime \prime} \rightarrow s$ can proceed. Consequently, all of $S$ can be constructed, and so each of the reactions $s \rightarrow a+b+c$ for all $s=\{a, b, c\} \in S$ can also now proceed. In summary, the reactants of all reactions in $\mathcal{R}_{(Y, S)}$ can be generated by starting just with molecules in $S^{\prime}$. Thus $\mathcal{R}_{(Y, S)}$ is $F^{\prime}$-generated for any subset $F^{\prime}=S^{\prime}$ of $F(=X)$ of size $q$ that provides an exact 3-cover for $Y$.

Conversely, suppose that $\mathcal{R}_{(Y, S)}$ is $F^{\prime}$-generated for some subset $F^{\prime}$ of $F(=X)$ of size at most $q=|Y|$ (we will show that this implies that $S$ contains an exact 3-cover). For each molecule $m$ in $F^{\prime}$ for which either:

(i) $m=y \in Y$, or

(ii) $m=w_{s} \in W$.

we proceed as follows. In case (i) select replace $y$ by $s$ for any $s \in S$ that contains $y$ (this is possible since we have assumed earlier, without loss of generality, that every element of $Y$ is present in at least one element of $S$ ); in case (ii) we replace $m$ by $s$ (i.e. the same $s$ appearing in $w_{s}$ ). Then $s$ generates $y$ in case (i), and $s$ generates the reactants required to generate $w_{s}$ in case (ii). In this way we can replace $F^{\prime}$ by a subset $S^{\prime}$ of $S$, with

$$
\left|S^{\prime}\right| \leq\left|F^{\prime}\right| \leq q
$$

and for which $\mathcal{R}_{(Y, S)}$ is $S^{\prime}$-generated. Thus, it is possible to generate each element of $Y$ by some series of reactions from $\mathcal{R}_{(Y, S)}$ starting with just the molecules in $S^{\prime}$.

Now, the only reactions that generate molecules in $Y$ are those that have a single reactant in $S$, and any $s \in S$ that is the product of any sequence of reactions from $\mathcal{R}_{(Y, S)}$ requires that all three elements of $s$ are either present or produced earlier in that sequence of reactions. It follows by an inductive argument that each molecule in $Y$ must be able to be generated by just a single reaction from $S^{\prime}$, 
and so $S^{\prime}$ is a 3 -cover for $Y$. This requires that $3\left|S^{\prime}\right| \geq|Y|$, and so $\left|S^{\prime}\right| \geq q$, which, combined with Inequality (1), gives $\left|S^{\prime}\right|=q$, and so (since $|Y|=3 q$ ) $S^{\prime}$ is an exact 3-cover for $Y$, as required.

\section{Additional file}

Additional file 1: Table S1. List of the 123 molecules of the food set and their impact on the RAF size.

\section{Competing interests}

The authors declare that they have no competing interests.

\section{Authors' contributions}

FLS and WH performed the bioinformatic analysis. MS constructed the mathematical proofs. FLS, WH, MS and WFM designed the research, analyzed the data, and wrote the paper. All authors read and approved the final manuscript.

\section{Acknowledgments}

This work was partially supported by the Allan Wilson Centre Molecular Ecology and Evolution to M.S., and by ERC Advanced Grant 232975 to W.F.M. We thank Eörs Száthmary and Martin Lercher for constructive comments on an earlier version of the manuscript.

\section{Author details}

${ }^{1}$ Institute of Molecular Evolution, Heinrich Heine Universität, Düsseldorf, Germany. ${ }^{2}$ SmartAnalytiX.com, Lausanne, Switzerland. ${ }^{3}$ Allan Wilson Centre Molecular Ecology and Evolution, University of Canterbury, Christchurch, New Zealand.

Received: 18 March 2014 Accepted: 27 November 2014 Published online: 01 April 2015

\section{References}

1. Ganti T. Organization of chemical reactions into dividing and metabolizing units: The chemotons. BioSystems. 1975;7(1):15-21.

2. Kauffman SA. Cellular homeostasis, epigenesis and replication in randomly aggregated macromolecular systems. J Cybern. 1971;1(1):71-96.

3. Kauffman SA. Autocatalytic sets of proteins. J Theor Biol. 1986;1 19:1-24.

4. Kauffman SA. The Origins of order. New York: Oxford University Press; 1993.

5. Vasas V, Fernando C, Santos M, Kauffman SA, Sathmáry E. Evolution before genes. Biol Direct. 2012;7:1.

6. Sievers D, von Kiedrowski G. Self-replication of complementary nucleotide-based oligomers. Nature. 1994;369:221-4.

7. Ashkenasy G, Jegasia R, Yadav M, Ghadiri MR. Design of a directed molecular network. Proc Nat Act Soc USA. 2004;101(30):10872-7.

8. Hayden EJ, von Kiedrowski G, Lehman N. Systems chemistry on ribozyme self-construction: Evidence for anabolic autocatalysis in a recombination network. Angew Chem Int Ed. 2008;120:8552-6.

9. Taran O, Thoennessen O, Achilles K, von Kiedrowski G. Synthesis of information-carrying polymers of mixed sequences from double stranded short deoxynucleotides. J Syst Chem. 2010;1 (9):9.

10. Vaidya N, Manapat ML, Chen IA, Xulvi-Brunet R, Hayden EJ, Lehman N. Spontaneous network formation among cooperative RNA replicators. Nature. 2012;491:72-7.

11. Kun A, Papp B, Szathmáry E. Computational identification of obligatorily autocatalytic replicators embedded in metabolic networks. Genome Biol. 2008;9(3):51.

12. Orth JD, Conrad TM, Na J, Lerman JA, Nam H, Feist AM, et al. A comprehensive genome-scale reconstruction of Escherichia coli metabolism-2011. Mol Syst Biol. 2011;7:535.

13. King GAM. Evolution of the coenzymes. BioSystems. 1980;13:23-45.

14. Dyson F. Origins of Life. New York: Cambridge University Press; 1985

15. Szathmáry E. The origin of the genetic code: amino acids as cofactors in an RNA world. Trends Genet. 1999:15:223-9.

16. Fernando C, Rowe J. Natural selection in chemical evolution. J Theor Biol. 2007:247:152-67.
17. Morowitz HJ, Srinivasan V, Smith E. Ligand field theory and the origin of life as an emergent feature of the periodic table of elements. Biol Bull. 2010;219:1-6.

18. Stockbridge RB, Lewis Jr CA, Yuan Y, Wolfenden R. Impact of temperature on the time required for the establishment of primordial biochemistry, and for the evolution of enzymes. Proc Nat Act Soc USA. 2010;107(51):22102-5

19. Wolfenden R. Benchmark reaction rates, the stability of biological molecules in water, and the evolution of catalytic power in enzymes. Annu Rev Biochem. 2011;80:645-67.

20. Zabinski RF, Toney MD. Metal ion inhibition of nonenzymatic pyridoxal phosphate catalyzed decarboxylation and transamination. J Am Chem Soc. 2001;123:193-8.

21. Russell MJ, Hall AJ. The emergence of life from iron monosulphide bubbles at a submarine hydrothermal redox and pH front. J Geol Soc. 1997; 154:377-402

22. Cody G. Transition metal sulfides and the origin of metabolism. Annu Rev Earth Planet Sci. 2004;32:569-99.

23. Wachtershauser G. Groundworks for an evolutionary biochemistry— the iron-sulfur world. Prog Biophys Mol Biol. 1992;58:85-201.

24. Steel $\mathrm{M}$. The emergence of a self-catalysing structure in abstract origin-of-life models. Appl Math Lett. 2000;3:91-5.

25. Hordijk W, Steel M. Detecting autocatalytic, self-sustaining sets in chemical reaction systems. J Theor Biol. 2004;227(4):451-61.

26. Hordijk W. Autocatalytic sets: From the origin of life to the economy. BioScience. 2013;63:877-81.

27. Hordijk W, Kauffman SA, Steel M. Required levels of catalysis for emergence of autocatalytic sets in models of chemical reaction systems. Int J Mol Sci. 2011;12(5):3085-101.

28. Hordijk W, Steel M, Kauffman S. The structure of autocatalytic sets: Evolvability, enablement, and emergence. Acta Biotheor. 2012;60(4): 379-92.

29. Mossel E, Steel M. Random biochemical networks: The probability of self-sustaining autocatalysis. J Theor Biol. 2005;233(3):327-36.

30. Hordijk W, Hein J, Steel M. Autocatalytic sets and the origin of life. Entropy. 2010;12(7):1733-42.

31. Hordijk W, Steel M. Predicting template-based catalysis rates in a simple catalytic reaction model. J Theor Biol. 2012;295:132-8

32. Hordijk W, Wills $P$, Steel M. Autocatalytic sets and biological specificity. Bull Math Biol. 2014;76(1):201-24.

33. Hordijk W, Hasenclever L, Gao J, Mincheva D, Hein J. An investigation into irreducible autocatalytic sets and power law distributed catalysis. Nat Comp. 2014;13:287-96.

34. Smith J, Steel M, Hordijk W. Autocatalytic sets in a partitioned biochemical network. J Syst Chem. 2014;5:2.

35. Steel M, Hordijk W, Smith J. Minimal autocatalytic networks. J Theor Biol. 2013;332:96-107.

36. Hordijk W, Steel M. A formal model of autocatalytic sets emerging in an RNA replicator system. J Syst Chem. 2013;4:3.

37. Magrane $M$, the UniProt Consortium. Uniprot knowledgebase: a hub of integrated protein data. Database (Oxford). 2011;2011:bar009.

38. Bagley RJ, Farmer JD, Fontana W. Evolution of a metabolism In: Langton CG, Taylor C, Farmer JD, Rasmussen S, editors. Artificial Life II. Redwood City: Addison-Wesley; 1992. p. 141-58.

39. Li F, Hinderberger J, Seedorf H, Zhang J, Buckel W, Thauer RK. Coupled ferredoxin and crotonyl coenzyme A (COA) reduction with $\mathrm{NADH}$ catalyzed by the butyryl-CoA dehydrogenasee/Etf complex from Clostridium kluyveri. J Bacteriol. 2008;190:843-50.

40. Mitchell P. Possible molecular mechanisms of the protonmotive function of cytochrome systems. J Theor Biol. 1976;62:327-67.

41. Sousa FL, Martin WF. Biochemical fossils of the ancient transition from geoenergetics to bioenergetics in prokaryotic one carbon compound metabolism. Biochem Biol Acta- Bioenergetics. 2014;1837:964-81.

42. Mackie A, Paley S, Sheare IM, Paulsen IT, Karp PD. Addition of Escherichia coli K-12 growth-observations and gene essentiality data to the EcoCyc database. J Bacteriol. 2014;196:982-8.

43. Kanehisa M, Goto S, Sato Y, Kawashima M, Furumichi M, Tanabe M. Data, information, knowledge and principle: back to metabolism in KEGG. Nucleic Acids Res. 2014:42:199-205.

44. Lane N, Martin WF. The origin of membrane bioenergetics. Cell. 2012;151(7):1406-16. 
45. Amend JP, Larowe DE, McCollom TM, Shock EL. The energetics of organic synthesis inside and outside the cell. Phil Trans R Soc B. 2013;368(1622):20120255.

46. Sousa FL, Thiergart T, Landan G, Nelson-Sathil S, Pereira IAC, Allen JF, et al. Early bioenergetic evolution. Phil Trans R Soc B. 2013;368(1622): 20130088.

47. Pál C, Papp B, Lercher MJ, Csermely P, Oliver SG, Hurst LD. Chance and necessity in the evolution of minimal metabolic networks. Nature. 2006;440:667-70.

48. Feist AM, Scholten JCM, Palsson B $\varnothing$, Brockman FJ, Ideker T. Modeling methanogenesis with a genome-scale metabolic reconstruction of Methanosarcina barkeri. Mol Syst Biol. 2006;2:4100046-114.

49. Becker SA, Palsson B $\varnothing$. Genome-scale reconstruction of the metabolic network in Staphylococcus aureus N315: an initial draft to the two-dimensional annotation. BMC Microbiol. 2005;5:8.

50. Lauhon CT, Kambampati R. The iscS gene in Escherichia coli is required for the biosynthesis of 4-thiouridine, thiamin, and NAD. J Biol Chem. 2000;275:20096-103.

51. Sprenger GA, Schörken U, Wiegert T, Grolle S, de Graaf AA, Taylor SV, et al. Identification of a thiamin-dependent synthase in E. coli required for the formation of the 1-deoxy-D-xylulose 5-phosphate precursor to isoprenoids, thiamin and pyridoxol. Proc Nat Act Soc USA. 1997;94: 12857-62.

52. Drewke C, Klein M, Clade D, Arenz A, R RM, Leistner E. 4-o-phosphoryl-L-threonine, a substrate of the $\mathrm{pdxC}(\operatorname{ser} \mathrm{C})$ gene product involved in vitamin B6 biosynthesis. FEBS Lett. 1996;22:179-82.

53. Reitzer L. Nitrogen assimilation and global regulation in Escherichia coli. Annu Rev Biochem. 2003;57:155-76.

54. Srinivasan $\mathrm{V}$, Morowitz HJ. Analysis of the intermediary metabolism of a reductive chemoautotroph. Biol Bull. 2009;217(3):222-32.

55. Martin WF, Russell MJ. On the origin of biochemistry at an alkaline hydrothermal vent. Phil Trans R Soc B. 2007;362:1887-925.

56. Kreuzer-Martin HW, Ehleringer JR, Hegg EL. Oxygen isotopes indicate most intracellular water in log-phase Escherichia coli is derived from metabolism. Proc Nat Act Soc USA. 2005;102:17337-41.

57. Baumler DJ, Peplinski RG, Reed JL, Glasner JD, Perna NT. The evolution of metabolic networks of E, coli. BMC Syst Biol. 2011;5:1-21.

58. Srinivasan V, Morowitz HJ. The canonical network of autotrophic intermediary metabolism: minimal metabolome of a reductive chemoautotroph. Biol Bull. 2009;216:126-30.

59. Handorf T, Ebenhoh O, Heinrich R. Expanding metabolic networks: Scopes of compounds, robustness, and evolution. J Mol Evol. 2005;61: 498-12.

60. Lane MD, Lynen F. The biochemical function of biotin, VI. Chemical structure of the carboxylated active site of propionyl carboxylase. Proc Nat Act Soc USA. 1963;49:379-85.

61. Bartoschek S, Vorholt JA, Thauer RK, Geierstanger BH, Griesinger C. $\mathrm{N}$-carboxymethanofuran (carbamate) formation from methanofuran and $\mathrm{CO}_{2}$ in methanogenic archaea. Thermodynamics and kinetics of the spontaneous reaction. Eur J Biochem. 2000;267:3130-8.

62. Thauer RK, Kaster AK, Seedorf H, Buckel W, Hedderich R. Methanogenic archaea: ecologically relevant differences in energy conservation. Nat Rev Microbiol. 2008;6:579-91.

63. Tawfik DS. Messy biology and the origins of evolutionary innovations. Nat Chem Biol. 2010;6:692-6.

64. Gerdes SY, Scholle MD, Campbell JW, Balázsi G, Ravasz E, Daugherty $M D$, et al. Experimental determination and system level analysis of essential genes in Escherichia coli MG1655. J Bacteriol. 2003;185:5673-84.

65. Baba T, Ara T, Hasegawa M, Takai Y, Okumura Y, Baba M, et al. Construction of Escherichia coli K-12 in-frame, single-gene knockout mutants: The Keio collection. Mol Syst Biol. 2006;2:1-11.

66. Papp B, Pál C, Hurst LD. Metabolic network analysis of the causes and evolution of enzyme dispensability in yeast. Nature. 2004;429(6992): $661-4$.

67. Amend JP, Shock EL. Energetics of amino acid synthesis in hydrothermal ecosystems. Science. 1998;281:1659-62.

68. Amend JP, McCollom TM. Energetics of biomolecule synthesis on early Earth. Chapter 4. New York: American Chemical Society; 2009, pp. 63-94.

69. Harold FM. The vital force - A study of bioenergetics. New York: WH Freeman; 1986.
70. Ma J, Katsonouri A, Gennis R. Subunit II of the cytochrome bo $\mathrm{b}_{3}$ ubiquinol oxidase from Escherichia coli is a lipoprotein. Biochem. 1997;36:11298-303.

71. Refojo PN, Sousa FL, Teixeira M, Pereira MM. The alternative complex III: a different architecture using known building modules. Biochem Biol Acta- Bioenergetics. 2010;1797:1869-76.

72. Oria-Hernández J, Riveros-Rosas H, Ramírez-Sílva L. Dichotomic phylogenetic tree of the pyruvate kinase family: $\mathrm{K}^{+}$-dependent and -independent enzymes. J Biol Chem. 2006;281:30717-724.

73. Zhang Y, Gladyshev VN. Comparative genomics of trace element dependence in biology. J Biol Chem. 2011;286:23623-9.

74. Dupont CL, Butcher A, Valas RE, Bourne PE, Caetano-Anollés G. History of biological metal utilization inferred through phylogenomic analysis of protein structures. Proc Nat Act Soc USA. 2010;107:10567-72.

75. Siebers B, Brinkmann H, Dörr C, Tjaden B, Lilie H, van der Oost J, et al. Archaeal fructose-1,6-bisphosphate aldolases constitute a new family of archaeal type Class I aldolase. J Biol Chem. 2001;276:28710-8.

76. Berry A, Marshall KE. Identification of zinc-binding ligands in the Class II fructose-1,6-bisphosphate aldolase of Escherichia coli. FEBS Lett. 1993;318: 11-6.

77. Thomson GJ, Howlett GJ, Ashcroft AE, Berry A. The dhnA gene of Escherichia coli encodes a Class I fructose bisphosphate aldolase. Biochem J. 1998;331:437-45.

78. Say RF, Fuchs G. Fructose 1,6-bisphosphate aldolase/phosphatase may be an ancestral gluconeogenic enzyme. Nat Lett. 2010;464:1077-81.

79. Meléndez-Hevia E, Isidoro A. The game of the pentose phosphate cycle. J Theor Biol. 1985;117:251-63.

80. Noor E, Eden E, Milo R, Alon U. Central carbon metabolism as a minimal biochemical walk between precursors for biomass and energy. Mol Cell. 2010;39:809-20.

81. Decker K, Jungermann K, Thauer RK. Energy production in anaerobic organisms. Angew Chem Int Ed. 1970;9:138-58.

82. Martin WF, Sousa FL, Lane N. Energy at life's origin. Science. 2014;344: 1092-3.

83. Palsson $B \varnothing$. Systems biology: properties of reconstructed networks. New York: Cambridge University Press; 2006.

84. Karp RM. Reducibility among combinatorial problems In: Miller RE, Thatcher JW, editors. Complexity of computer computations. New York: Plenum; 1972. p. 85-103.

\section{Publish with ChemistryCentral and every scientist can read your work free of charge \\ “Open access provides opportunities to our colleagues in other parts of the globe, by allowing anyone to view the content free of charge." \\ W. Jeffery Hurst, The Hershey Company.}

- available free of charge to the entire scientific community

- peer reviewed and published immediately upon acceptance

- cited in PubMed and archived on PubMed Central

- yours - you keep the copyright

Submit your manuscript here:

http://www.chemistrycentral.com/manuscript/<smiles>c1ccccc1</smiles>

Chemistry Central 